\title{
Synchronization of globally-coupled phase oscillators: singularities and scaling for general couplings
}

\author{
John David Crawford \\ Department of Physics and Astronomy \\ University of Pittsburgh \\ Pittsburgh, PA 15260 \\ K.T.R. Davies \\ Department of Physics \\ Duquesne University \\ Pittsburgh, Pennsylvania 15282-1503
}

\begin{abstract}
The onset of collective behavior in a population of globally coupled oscillators with randomly distributed frequencies is studied for phase dynamical models with arbitrary coupling; the effect of a stochastic temporal variation in the frequencies is also included. The Fokker-Planck equation for the coupled Langevin system is reduced to a kinetic equation for the oscillator distribution function. Instabilities of the phase-incoherent state are studied by center manifold reduction to the amplitude dynamics of the unstable modes. Depending on the coupling, the coefficients in the normal form can be singular in the limit of weak instability when the diffusive effect of the noise is neglected. A detailed analysis of these singularities to all orders in the normal form expansion is presented. Physically, the singularities are interpreted as predicting an altered scaling of the entrained component near the onset of synchronization.
\end{abstract}

keywords: oscillators, bifurcation, symmetry

January 9, 1997 


\section{Contents}

\begin{tabular}{lll}
\hline 1 & Introduction & 1
\end{tabular}

\begin{tabular}{|lrr}
\hline 2 & Linear analysis & 8
\end{tabular}

3 Amplitude equation on the unstable manifold 15

3.1 Analysis of $H\left(\theta, \omega, \alpha, \alpha^{*}\right) \ldots \ldots \ldots \ldots \ldots$. . . . . . . . . . . . . . . . . .

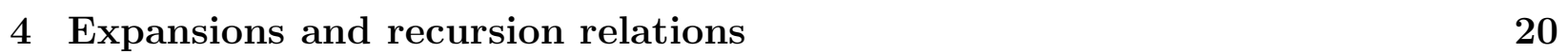

4.1 Recursion relations for $I_{m, j} \ldots \ldots \ldots \ldots$. . . . . . . . . . . . 22

5 Pinching singularities at low order $\quad 24$

$5.1 \quad$ Evaluation of the coefficient $p_{1} \ldots \ldots \ldots$. . . . . . . . . . . . . . . . . 24

5.2 The fifth order coefficient $p_{2} \ldots \ldots \ldots \ldots$. . . . . . . . . . 27

$6 \quad$ Singularity structure of the expansion to all orders 29

6.1 Definition of the index . . . . . . . . . . . . . . . . . . 29

6.2 Calculation of the index . . . . . . . . . . . . . . . . . . . . . . . . 34

6.3 The main result . . . . . . . . . . . . . . . . . . . . . . . . . . . . . . . . . 37

6.4 Implications of Theorem 6.1 . . . . . . . . . . . . . . . . . . . . . . . . 43

7 Special Cases: $f_{2 l}=0 \quad 45$

7.1 Absence of the cubic singularity: $f_{2 l}=0$ and $f_{3 l} \neq 0$. . . . . . . . . . 45

7.2 Implications for scaling . . . . . . . . . . . . . . . . . . . . . . . 47

7.3 Single component couplings . . . . . . . . . . . . . . . . . . . . . . . . . . . 47

8 Evaluation of Daido's order function 4

\begin{tabular}{lll}
\hline 9 & Discussion & 51
\end{tabular}

\begin{tabular}{lll}
\hline 10 & Acknowledgements & 53
\end{tabular}

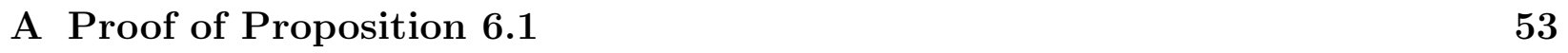

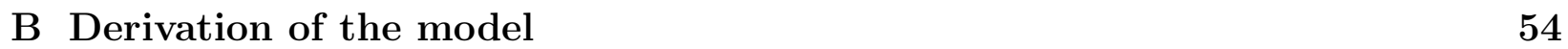

\section{Introduction}

A phase dynamics description of $N$ limit cycle oscillators is feasible for sufficiently weak interaction. [1, 2, 3] The uncoupled system is attracted to an N-torus and for weak coupling 
this attracting torus will persist; the oscillator phases $\left(\theta_{1}(t), \ldots, \theta_{N}(t)\right)$ are coordinates on the perturbed torus. In this paper we study such systems where the interaction is mediated by a global coupling. [2, 3] More precisely, we consider models of the form,

$$
\dot{\theta}_{i}=\omega_{i}+\frac{K}{N} \sum_{j=1}^{N} f\left(\theta_{j}-\theta_{i}\right)+\xi_{i}(t)
$$

with the frequencies $\omega_{i}$ of the uncoupled oscillators drawn from a distribution $g(\omega)$ characteristic of the population or array. For both physical and mathematical reasons, it is interesting to include in (1) the effect of extrinsic white noise $\xi_{i}(t)$ perturbing the deterministic phase dynamics; this term is defined by the ensemble averages $\left\langle\xi_{i}(t)\right\rangle=0$ and $<\xi_{i}(s) \xi_{j}(t)>=2 D \delta_{i j} \delta(s-t)$. When the oscillators are uncoupled $(K=0)$, then the noise and the intrinsic variation in the frequencies spreads the population in phase. For suitably chosen coupling functions $f(\phi)$ as the strength of the coupling $K$ increases, the instantaneous frequencies $\dot{\theta}_{i}$ may become entrained and the phase disorder of the uncoupled system gives way to an entrained state with partial or total phase coherence.

The collective oscillations produced by such frequency-entrained states is of interest in physics, chemistry, biology and rather recently neuroscience.[2, 田, 5, 6, 7] The phase dynamics model (1) was popularized especially by Kuramoto and his collaborators for the coupling $f(\phi)=\sin \phi$ since this choice allowed a mathematically tractable description of the onset of entrainment. [2] For a coupling $f(\phi)$ of arbitrary form, the model is a special case of a general normal form obtained by averaging.[3] Very recently, the averaged equations for a series array of weakly interacting Josephson junctions has been shown to have the form (四) with $f(\phi)=\sin \phi$ at leading order in the coupling strength $K$. [8] This result extended similar studies that had treated the junctions as identical oscillators. [9, 10,

Most research on phase oscillators with global couplings has been motivated by their appeal as theoretical models of synchronization rather than as realistic descriptions of specific 
experimental systems. In this literature, until very recently, the preoccupation with the simple coupling function considered by Kuramoto has been nearly univeral; see [6, 11] for discussions with many references. Nevertheless, couplings $f(\phi)$ of more general form are of interest for several reasons. First, interactions that are derived when a reduction to phase dynamics is actually carried out can easily have a more complicated structure. [2, 12] Secondly, recent results indicate that when the form of $f(\phi)$ is generalized, the emergence of the entrained state can have qualitative different features. 13, 14, 15] More precisely, if $K_{c}$ is the critical coupling, above which the synchronized state appears, then the "size" of the entrained component scales like $\left(K-K_{c}\right)^{\beta}$ above threshold. In the absence of noise, Daido discovered $\beta=1$ when $f(\phi)$ contains a second harmonic such as $\sin 2 \phi$, but for the Kuramoto model the exponent drops to $\beta=1 / 2$, i.e. the entrainment for the more general coupling can be much weaker at the same coupling stength $K$.13 In this paper, we present a detailed analysis of the first transistion to a state with frequency entrainment that illuminates the interplay between the intrinsic disorder $g(\omega)$, the external noise, and the coupling in determining the exponent $\beta$. In particular, we make no further restrictions on $f(\phi)$ other than to assume it has a Fourier expansion

$$
f(\phi)=\sum_{n=-\infty}^{\infty} f_{n} e^{i n \phi} .
$$

For large oscillator populations, it is natural to analyze the system in the limit $N \rightarrow \infty$ because of the relative simplicity afforded by a continuum description of the population using a density function $\rho(\theta, \omega, t)$. A convenient definition divides out the frequency dependence of the uncoupled system so that $g(\omega) \rho(\theta, \omega, t) d \theta d \omega$ describes the fraction of oscillators with natural frequency in $[\omega, \omega+d \omega]$ and phase in $[\theta, \theta+d \theta]$, and $\rho(\theta, \omega, t) d \theta$ describes the fraction of oscillators at natural frequency $\omega$ with phase in $[\theta, \theta+d \theta]$. This latter interpretation fixes the normalization $\int \rho(\theta, \omega, t) d \theta=1$. The dynamics of the system (11) is formulated as a 
kinetic equation for $\rho(\theta, \omega, t)$,

$$
\frac{\partial \rho}{\partial t}+\frac{\partial(\rho v)}{\partial \theta}=D \frac{\partial^{2} \rho}{\partial \theta^{2}}
$$

with the deterministic part of the phase velocity (11) expressed as an integral over the population

$$
v(\theta, \omega, t)=\omega+K \int_{0}^{2 \pi} d \theta^{\prime} \int_{-\infty}^{\infty} d \omega^{\prime} f\left(\theta^{\prime}-\theta\right) \rho\left(\theta^{\prime}, \omega^{\prime}, t\right) g\left(\omega^{\prime}\right) .
$$

This equation has been applied recently to the Kuramoto model; 16 -19 our application to (1]) for arbitrary coupling is a generalization of the results for $f(\phi)=\sin \phi$. [17, 19] For completeness, the derivation of (3) - (4) is outlined in Appendix B.

The kinetic equation has a special solution,

$$
\rho=\rho_{0} \equiv \frac{1}{2 \pi}
$$

which describes a population of oscillators spread out unpreferentially in phase. This uniform or incoherent distribution is an equilibrium since $v(\theta, \omega)=\omega+K f_{0}$ for $\rho=\rho_{0}$ implies $\partial_{t} \rho=0$. The occurrence of instabilities of $\rho_{0}$ leading to states with nontrivial phase dependence is established by a linear stability analysis of $\rho_{0}$ in section 1 and a nonlinear analysis of the instabilities in sections $3-7$. The nonlinear analysis focuses on the evolution of the linearly unstable mode described by the flow on the unstable manifold of the equilibrium; the amplitude equation describing this flow are formulated and studied in general terms in section 3. The detailed calculation of the unstable manifold flow given in section 4 relies on expansions in the amplitude of the unstable mode. The exponent $\beta$ emerges as the scaling exponent required to balance the linear instability against the nonlinear terms in the amplitude equation. In the regime of weak linear instability, different exponents can arise because the behavior of the nonlinear terms varies considerably depending on the form of the coupling and strength of the external noise. These features of the expansion are studied in sections 5 - 0 . 
Our conclusions regarding the properties of the amplitude equation are valid to all orders in the expansion. This level of generality is possible in part because the recursion relations for the expansion are considerably simplified by the symmetry of the problem. The model (8) - (4) has $\mathrm{SO}(2)$ or $\mathrm{O}(2)$ symmetry depending on details of the population and the coupling. The group $\mathrm{O}(2)$ is generated by rotations $\beta \cdot(\theta, \omega)=(\theta+\beta, \omega)$ and reflections $\kappa \cdot(\theta, \omega)=$ $-(\theta, \omega)$ which act on functions $\rho(\theta, \omega)$ in the usual way: for any transformation $\chi \in \mathrm{O}(2)$, $(\chi \cdot \rho)(\theta, \omega) \equiv \rho\left(\chi^{-1} \cdot(\theta, \omega)\right)$. The dynamics (3) - (四) is equivariant with respect to rotations for arbitrary choices of $g(\omega)$ and $f(\phi)$; if, in addition, $g(\omega)=g(-\omega)$ and $f(\phi)=-f(-\phi)$, then the system commutes with the reflection $\kappa$ as well. In the latter circumstance the model has $\mathrm{O}(2)$ symmetry, otherwise the rotational symmetry alone corresponds to $\mathrm{SO}(2)$. Note that the mean frequency of $g(\omega)$ can always be shifted to zero and we assume that this has been done.

The onset of linear instability for the incoherent state $\rho_{0}$ has some unusual features, especially in the limit of weak noise $D \rightarrow 0$. This was first noticed in the context of the Kuramoto model, [17]- [19] and is emphasized in the linear analysis of section 2. In the absence of noise, the unstable modes correspond to eigenvalues emerging from a neutral continuum at onset; thus, the standard framework of center manifold reduction cannot be directly applied. We proceed by first deriving the amplitude equation on the center-unstable manifold with $D>0$, in this case the diffusion term (3) shifts the continuous spectrum off the imaginary axis and the center manifold reduction is straightforward. [20, 21] The resulting amplitude equation is then examined by first reducing the noise $D \rightarrow 0$ and then letting the linear growth rate of the mode approach zero $\gamma \rightarrow 0^{+}$. In this way, we start with an amplitude equation on the unstable manifold and examine its features as the unstable eigenvalues are allowed to approach a neutral continuous spectrum on the imaginary axis.

Our procedure for extracting $\beta$ from the amplitude equation can be illustrated by a 
simple example. Let $A(t)$ denote the mode amplitude satisfying

$$
\dot{A}(t)=\gamma A+a_{1}(\gamma) A^{3}+a_{2}(\gamma) A^{5}+\cdots
$$

where the nonlinear coefficients $a_{1}, a_{2}, \ldots$ depend on the parameters of the problem such as the growth rate $\gamma$. For small $\gamma$, this equation should be viewed as a singular perturbation problem with the linear term $\gamma A$ representing the perturbation. If $\gamma>0$, then there is always a neighborhood of the equilibrium $A=0$ where the perturbation dominates the unperturbed system and can completely change the dynamics. As in other such singular problems, a possible strategy is to seek a (singular) change of variables which transforms (6) into a regular perturbation problem. Thus it is natural to define a new amplitude by

$$
A(t)=\gamma^{\beta} r\left(\gamma^{\delta} t\right)
$$

and rewrite (6) in terms of $r(\tau)$

$$
\frac{d r}{d \tau}=\gamma^{1-\delta} r(\tau)+\gamma^{2 \beta-\delta} a_{1}(\gamma) r^{3}+\gamma^{4 \beta-\delta} a_{2}(\gamma) r^{5}+\cdots
$$

If possible, the choice of $\beta$ and $\delta$ should be made so that each term in (8) is well behaved as $\gamma \rightarrow 0^{+}$, and furthermore so that the effect of $\gamma>0$ is a regular perturbation of the system at $\gamma=0$. The standard values are of course $\beta=1 / 2$ and $\delta=1$ yielding

$$
\frac{d r}{d \tau}=r(\tau)+a_{1}(\gamma) r^{3}+\gamma a_{2}(\gamma) r^{5}+\cdots
$$

The unperturbed system $\dot{r}=r+a_{1}(0) r^{3}$ now includes the linear term and near $r=0$ the effect of small $\gamma$ is unimportant. The change of variables (7) is singular at $\gamma=0$ as expected, but nevertheless we have determined the overall scaling $\beta=1 / 2$ for nonlinear solutions near the equilibrium in the regime of weak instability. In addition, since terms in (8) at fifth order or higher are at least $\mathcal{O}(\gamma)$, the unperturbed problem truncates to a simple balance between the linear instability and the dominant nonlinear term. Note that since $\gamma=0$ at $K=K_{c}$, 
then just above threshold $\gamma \sim\left(K-K_{c}\right)$, and the definition in () implies $|A| \sim\left(K-K_{c}\right)^{\beta}$ for the scaling of the mode ampltude near onset.

The preceeding discussion contains the unstated assumption that the coefficients $a_{1}, a_{2}, \ldots$ have well-defined finite limits as $\gamma \rightarrow 0$. In the phase dynamical model (3) - (田) this assumption is correct for any choice of $f(\phi)$ and $g(\omega)$ as long as $D>0$. However if we set $D=0$ before taking the limit $\gamma \rightarrow 0^{+}$, then the nonlinear coefficients are singular in general although the details depend on $f(\phi)$. These singularities require a different choice for $\beta$ to accomplish the desiderata enumerated above. It turns out that when one has a coupling with only a single Fourier component such as in the Kuramoto model then the expansion coefficients are nonsingular to all orders. Thus single component couplings are special and very atypical. The most typical or generic behavior is $\beta=1$ as was found by Daido in the examples he considered. [13, 15] We further discuss the relation between our approach and his theory in section 8 .

A key aspect of the singular behavior encountered in this problem is the emergence of the critical eigenvalues from a neutrally stable continuous spectrum. It has become clear recently that this characteristic is shared by a diverse set of additional examples which include the onset of linear instability in collisionless plasma 22 24 and in fluid shear flows 25 [26], instabilities of solitary waves 27]-29], and bifurcations in "mean field" descriptions of the dynamics of bubble clouds in fluids 30. Aside from the present work, only for the collisionless plasma models have the resulting singularities in the amplitude expansion been fully analyzed. Our proofs in this paper are adapted from the techniques used in 23. 


\section{Linear analysis}

Let $\eta \equiv \rho(\theta, \omega, t)-\rho_{0}$ denote the deviation from $\rho_{0}$, and rewrite the dynamics (3) - (四) to describe the evolution of $\eta$ :

$$
\frac{\partial \eta}{\partial t}=\mathcal{L} \eta+\mathcal{N}(\eta)
$$

where the linear and nonlinear operators are defined by

$$
\begin{gathered}
\mathcal{L} \eta=D \frac{\partial^{2} \eta}{\partial \theta^{2}}-\left(\omega+K f_{0}\right) \frac{\partial \eta}{\partial \theta}+\frac{K}{2 \pi} \int_{0}^{2 \pi} d \theta^{\prime} \int_{-\infty}^{\infty} d \omega^{\prime} f^{\prime}\left(\theta^{\prime}-\theta\right) \eta\left(\theta^{\prime}, \omega^{\prime}, t\right) g\left(\omega^{\prime}\right) \\
\mathcal{N}(\eta)=K \int_{0}^{2 \pi} d \theta^{\prime} \int_{-\infty}^{\infty} d \omega^{\prime} \eta\left(\theta^{\prime}, \omega^{\prime}, t\right) g\left(\omega^{\prime}\right)\left[\eta(\theta, \omega, t) f^{\prime}\left(\theta^{\prime}-\theta\right)\right. \\
\left.-\frac{\partial \eta}{\partial \theta}(\theta, \omega, t) f\left(\theta^{\prime}-\theta\right)\right] .
\end{gathered}
$$

In (11) - (12), $f^{\prime}(\phi) \equiv d f / d \phi$, and note that the normalization of $\rho$ implies

$$
\int_{0}^{2 \pi} d \theta \eta(\theta, \omega, t)=0
$$

The equilibrium $\rho_{0}$ is invariant under translations $\beta$ and reflections $\kappa$; hence the dynamics for $\eta$ has the same symmetries as the original system. This can be verified explicitly by noting that the operators $\mathcal{L}$ and $\mathcal{N}$ commute with rotations for arbitrary choices of $g(\omega)$ and $f(\phi)$, and also commute with $\kappa$ when $g(\omega)=g(-\omega)$ and $f(\phi)=-f(-\phi)$.

Since $\rho_{0}$ is independent of $\theta$, the eigenfunctions $\mathcal{L} \Psi=\lambda \Psi$ have the form

$$
\Psi(\theta, \omega)=\psi(\omega) e^{i n \theta}
$$

with $\psi(\omega)$ required to satisfy the eigenvalue equation

$$
L_{n} \psi=\lambda \psi
$$

where

$$
L_{n} \psi \equiv-i n\left[\left(\omega+K f_{0}-i n D\right) \psi+K f_{n}^{*} \int_{-\infty}^{\infty} d \omega^{\prime} g\left(\omega^{\prime}\right) \psi\left(\omega^{\prime}\right)\right]
$$


Because the $n=0$ component of $\eta$ is zero (13), we can restrict our consideration of (15) to $n \neq 0$.

The spectrum of $L_{n}$ corresponds to values of $\lambda$ where the resolvent $R_{n}(\lambda) \equiv\left(\lambda-L_{n}\right)^{-1}$ fails to be a bounded operator. By solving the equation $\left(\lambda-L_{n}\right) \hat{\phi}=\phi$ for $\hat{\phi}(\omega)$ we obtain the resolvent explicitly, i.e. $\hat{\phi}=R_{n}(\lambda) \phi$ with

$$
R_{n}(\lambda) \phi=\frac{-i / n}{\omega+K f_{0}-i \lambda / n-i n D}\left[\phi(\omega)-\frac{K f_{n}^{*}}{\Lambda_{n}(i \lambda / n)} \int_{-\infty}^{\infty} \frac{d \omega^{\prime} g\left(\omega^{\prime}\right) \phi\left(\omega^{\prime}\right)}{\omega^{\prime}+K f_{0}-i \lambda / n-i n D}\right]
$$

where, in the second term, $\Lambda_{n}(z)$ denotes

$$
\Lambda_{n}(z) \equiv 1+K f_{n}^{*} \int_{-\infty}^{\infty} d \omega \frac{g(\omega)}{\omega+K f_{0}-z-i n D}
$$

The details of a completely rigorous discussion of $R_{n}(\lambda)$ will depend of course on the choice of a specific function space for the problem, e.g. square integrable functions of $\omega$. For our purposes, it suffices to ignore these technicalities and discuss the spectrum of $L_{n}$ carefully though heuristically.

There are two ways $R_{n}(\lambda) \phi$ can be singular: when $i \lambda / n$ hits a zero of the function $\Lambda_{n}$ and when the denominator in (17) vanishes, i.e. when $\lambda=-i n\left(\omega+K f_{0}-i n D\right)$. Since $-\infty<\omega<\infty$, this latter set of values coincides with the line $\operatorname{Re}(\lambda)=-n^{2} D$, while the first set of values are typically discrete $\lambda=-i n z_{j}$ since $\Lambda_{n}\left(z_{j}\right)=0$ will have discrete solutions $\left\{z_{1}, z_{2}, \ldots\right\}$. These two components generally correspond to the essential spectrum and the point spectrum (eigenvalues), respectively.

This distinction is clarified by solving the eigenvalue equation directly. Let $\lambda=-i n z$, then (15) reads

$$
\left(\omega+K f_{0}-z-i n D\right) \psi(\omega)=-K f_{n}^{*} \int_{-\infty}^{\infty} d \omega^{\prime} g\left(\omega^{\prime}\right) \psi\left(\omega^{\prime}\right)
$$

The possible solutions for $\operatorname{Im}(z) \neq-n D$ and $\operatorname{Im}(z)=-n D$ are quite different and we discuss them separately. 
For $\operatorname{Im}(z) \neq-n D$ or $\operatorname{Re}(\lambda) \neq-n^{2} D$, we can divide by $\left(\omega+K f_{0}-z-i n D\right)$ and obtain the form of the eigenfunction

$$
\psi(\omega)=\frac{-K f_{n}^{*} \int d \omega^{\prime} g\left(\omega^{\prime}\right) \psi\left(\omega^{\prime}\right)}{\omega+K f_{0}-z-i n D}
$$

in terms of the constant $\int d \omega^{\prime} g \psi$. A nontrivial solution in (20) is found only if this constant is non-zero, in which case we can adopt the normalization

$$
\int_{-\infty}^{\infty} d \omega^{\prime} g\left(\omega^{\prime}\right) \psi\left(\omega^{\prime}\right)=1
$$

Together (20) and (21) determine an eigenfunction

$$
\psi(\omega)=\frac{-K f_{n}^{*}}{\omega+K f_{0}-z-i n D}
$$

provided the normalization condition (21) for $\psi$ can be satisfied self-consistently. This requirement is precisely $\Lambda_{n}(z)=\Lambda_{n}(i \lambda / n)=0$. Thus a root $\Lambda_{n}(z)=0$ implies the existence of an eigenvalue $\lambda=-i n z$ with eigenfunction (22).

For all choices of $g(\omega)$ and $f(\phi), \Lambda_{n}(z)$ satisfies the identity

$$
\Lambda_{n}(z)^{*}=\Lambda_{-n}\left(z^{*}\right)
$$

in addition, with reflection symmetry $f(\phi)=-f(-\phi)$ and $g(\omega)=g(-\omega)$, a further identity holds

$$
\Lambda_{n}(z)=\Lambda_{-n}(-z)
$$

The first identity implies that complex eigenvalues must come in conjugate pairs $\left(\lambda, \lambda^{*}\right)$ with conjugate eigenvectors $\Psi(\theta, \omega)$ and $\Psi(\theta, \omega)^{*}$, respectively. For a real eigenvalue $(\operatorname{Re}(z)=0)$, the first identity reduces to the second (24) and implies that the eigenvalue will always have (at least) two linearly independent eigenvectors, $\Psi$ and $\Psi^{*}$. When we have a complex eigenvalue for a reflection symmetric population, then (24) holds and again there will be two 
linearly independent eigenvectors $\Psi(\theta, \omega)$ and $\Psi(-\theta,-\omega)$. Without reflection symmetry, the typical case is a complex eigenvalue, but with reflection symmetry both real and complex eigenvalues can occur generically.

The actual occurrence of roots for $\Lambda_{n}(z)$ is a detailed question depending on the frequency distribution $g$ and the coupling; examples have been found for which $\Lambda_{n}(z)$ has no roots. 117 In the complex $z$ plane, $\Lambda_{n}(z)$ is an analytic function for $\operatorname{Im}(z) \neq-n D$ with a discontinuity across $\operatorname{Im}(z)=-n D$ whenever $g\left(\operatorname{Re}(z)-K f_{0}\right) \neq 0$. The discontinuity signals a branch cut for $\Lambda_{n}(z)$ and the magnitude of the discontinuity is given by the boundary values at $\operatorname{Re}(z)=r:$

$$
\lim _{\epsilon \rightarrow 0^{+}} \Lambda_{n}(r-i n D \pm i \epsilon)=1+K f_{n}^{*}\left[\mathrm{PV} \int_{-\infty}^{\infty} d \omega \frac{g(\omega)}{\omega+K f_{0}-r}\right] \pm i \pi K f_{n}^{*} g\left(r-K f_{0}\right) .
$$

As the parameters of the coupling or frequency distribution $g$ are varied, the roots of $\Lambda_{n}(z)$ generally change. In particular, roots can appear or disappear at the branch cut.

When $D=0$, the appearance of a root coincides with the onset of linear instability and the boundary values (25) provide closed form expressions for the coupling $K_{c}$ and frequency $\Omega_{c}=-\operatorname{Im}(\lambda)$ at criticality. Let $z_{0}=(\Omega+i \gamma) / n$ denote the root with $\gamma>0$, and $0=$ $\Lambda_{n}((\Omega+i \gamma) / n)$. As $\gamma \rightarrow 0^{+}$, we obtain

$$
0=1+K_{c} f_{n}^{*}\left[\mathrm{PV} \int_{-\infty}^{\infty} d \omega \frac{g(\omega)}{\omega-\Omega^{\prime}}\right]+i \pi K_{c} f_{n}^{*} g\left(\Omega^{\prime}\right) .
$$

where $\Omega^{\prime}=\Omega_{c} / n-K_{c} f_{0}$. The real and imaginary parts of this equation must vanish separately and this implies

$$
\begin{aligned}
\frac{-\operatorname{Im}\left(f_{n}\right)}{\pi\left|f_{n}\right|^{2} g\left(\Omega^{\prime}\right)} & =K_{c} \\
\frac{\pi \operatorname{Re}\left(f_{n}\right) g\left(\Omega^{\prime}\right)}{\operatorname{Im}\left(f_{n}\right)} & =\int_{0}^{\infty} \frac{d \Delta}{\Delta}\left[g\left(\Omega^{\prime}+\Delta\right)-g\left(\Omega^{\prime}-\Delta\right)\right] .
\end{aligned}
$$


The second equation determines $\Omega^{\prime}$ and then the first equation determines $K_{c}$; for $n=1$ and $f_{0}=0$ these expressions reduce to the formulas derived by Daido in his order function analysis. 31]

For $\operatorname{Im}(z)=-n D$ or $\operatorname{Re}(\lambda)=-n^{2} D$, the eigenvalue equation (19) has no nonsingular solutions. One can introduce distributions as solutions following the closely related spectral theory for the Vlasov equation, but we shall not require this development for our study (cf. [32] for details).

In general, the spectrum of $\mathcal{L}$ has both eigenvalues and a continuous component: for each mode number $n=1,2, \ldots$, there is a line of continuous spectrum at $\operatorname{Re} \lambda=-n^{2} D$; in addition $\mathcal{L}$ has eigenvalues when the function $\Lambda_{n}$ has roots. We are specifically interested in eigenvalues that cross the imaginary axis, signifying instabilities of $\rho_{0}$. Depending on the coupling, this can occur for any Fourier component $n=l$, provided there is a root $\Lambda_{l}\left(z_{0}\right)=0$ such that $\operatorname{Im}\left(z_{0}\right)$ becomes positive. Henceforth $l$ and $z_{0}$ refer to the eigenvalue $\lambda=-i l z_{0}$ determining the instability and the unstable eigenfunction is

$$
\begin{aligned}
\Psi(\theta, \omega) & =\psi(\omega) e^{i l \theta} \\
\psi(\omega) & =\frac{-K f_{l}^{*}}{\omega+K f_{0}-z_{0}-i l D} .
\end{aligned}
$$

Clearly the coupling must have a Fourier component $f_{l} \neq 0$.

We further restrict the problem with two additional assumptions. First, the root $z_{0}$ is simple, i.e. $\Lambda_{l}^{\prime}\left(z_{0}\right) \neq 0$ where $\Lambda_{l}^{\prime} \equiv d \Lambda_{l} / d z$; secondly, the center subspace at criticality is two-dimensional, in particular, all other roots of $\Lambda_{n}$ for any $n>0$ have negative imaginary parts and remain bounded away from the real axis. The first assumption ensures that there are no generalized eigenvectors; the second restricts the analysis to populations with rotation symmetry $(\mathrm{SO}(2))$ and complex eigenvalues, or populations with rotation and reflection symmetry $(\mathrm{O}(2))$ and a real eigenvalue. [19] This restriction excludes one possible 
codimension-one bifurcation, a Hopf bifurcation with $\mathrm{O}(2)$ symmetry, which involves a fourdimensional center subspace; a discussion of $O(2)$ Hopf for the Kuramoto model has been given elsewhere. 19.

The projection of $\eta$ along the critical mode (30) requires an inner product

$$
(A, B) \equiv \int_{0}^{2 \pi} d \theta \int_{-\infty}^{\infty} d \omega A(\theta, \omega)^{*} B(\theta, \omega)
$$

so that we can define an adjoint operator for $\mathcal{L}$

$$
\left(\mathcal{L}^{\dagger} A, B\right)=(A, \mathcal{L} B)
$$

In terms of the Fourier expansion $A=\sum_{n} A_{n} \exp (i n \theta)$, the inner product becomes $(A, B)=$ $2 \pi \sum_{n}<A_{n}, B_{n}>$ using a convenient notation for the $\omega$ integration

$$
<A_{n}, B_{n}>\equiv \int_{-\infty}^{\infty} d \omega A_{n}(\omega)^{*} B_{n}(\omega)
$$

A simple calculation shows that

$$
\mathcal{L}^{\dagger} A=\sum_{n=-\infty}^{\infty} e^{i n \theta} L_{n}^{\dagger} A_{n}(\omega)
$$

with

$$
\left(L_{n}^{\dagger} A_{n}\right)(\omega)=i n\left[\left(\omega+K f_{0}+i n D\right) A_{n}(\omega)+K f_{n} g(\omega) \int_{-\infty}^{\infty} d \omega^{\prime} A_{n}\left(\omega^{\prime}\right)\right] .
$$

The discussion of the spectrum of $L_{n}^{\dagger}$ parallels the analysis for $L_{n}$, and we simply state the results we shall require. The root $\Lambda_{l}\left(z_{0}\right)=0$ implies an eigenfunction $\mathcal{L}^{\dagger} \tilde{\Psi}=\lambda^{*} \tilde{\Psi}$ where

$$
\begin{aligned}
\tilde{\Psi}(\theta, \omega) & =\frac{1}{2 \pi} \tilde{\psi}(\omega) e^{i l \theta} \\
\tilde{\psi}(\omega) & =\frac{-g(\omega)}{\Lambda_{l}^{\prime}\left(z_{0}\right)^{*}\left(\omega+K f_{0}-z_{0}^{*}+i l D\right)} .
\end{aligned}
$$

This adjoint eigenfunction satisfies $(\tilde{\Psi}, \Psi)=1$ and defines the projection, $\eta \rightarrow(\tilde{\Psi}, \eta) \Psi$, from $\eta$ onto the $\Psi$ component of $\eta$. This projection is the only aspect of the adjoint theory that we need. 
Before developing the nonlinear theory, we illustrate our discussion with a simple example of the instabilities we intend to analyze. Let the oscillator population be described by a Lorentzian frequency distribution,

$$
g(\omega)=\frac{\Delta}{\pi}\left[\frac{1}{\omega^{2}+\Delta^{2}}\right],
$$

then for $\operatorname{Im}(z) \neq-l D, \Lambda_{l}(z)$ is easily evaluated by contour integration to obtain

$$
\Lambda_{l}(z)= \begin{cases}\frac{z-K\left(f_{0}+f_{l}^{*}\right)+i(l D-\Delta)}{z-K f_{0}+i(l D-\Delta)} & \operatorname{Im}(z)<-l D \\ \frac{z-K\left(f_{0}+f_{l}^{*}\right)+i(l D+\Delta)}{z-K f_{0}+i(l D+\Delta)} & \operatorname{Im}(z)>-l D .\end{cases}
$$

For $\operatorname{Im}(z)<-l D$, there is a root at $z_{s}=K\left(f_{0}+f_{l}^{*}\right)-i(l D-\Delta)$ when the coupling satisfies $K \operatorname{Im}\left(f_{l}\right)>\Delta$. The eigenvalues $\lambda=-i l z_{s}$ have negative real parts and fall to the left of the continuous spectrum at $\operatorname{Re} \lambda=-l^{2} D$. An eigenvalue sitting to the right of the continuous spectrum requires a root with $\operatorname{Im}(z)>-l D$; for this example $\Lambda_{l}(z)$ has one such root at $z_{u}=K\left(f_{0}+f_{l}^{*}\right)-i(l D+\Delta)$ when the coupling satisfies $K \operatorname{Im}\left(f_{l}\right)<-\Delta$. This root yields the eigenvalue

$$
\lambda=-i l z_{u}=\gamma-i \Omega,
$$

where

$$
\begin{aligned}
\gamma & =-l\left[l D+\Delta+K \operatorname{Im}\left(f_{l}\right)\right] \\
\Omega & =l K\left[\operatorname{Re}\left(f_{l}\right)+f_{0}\right] .
\end{aligned}
$$

These modes are linearly stable for $l D+\Delta+K \operatorname{Im}\left(f_{l}\right)>0$ and become linearly unstable for $K>K_{c}$ where $K_{c}=-(l D+\Delta) / \operatorname{Im}\left(f_{l}\right)$. Note, that we find eigenvalues of $L_{l}$ only when $\left|K \operatorname{Im}\left(f_{l}\right)\right|>\Delta ;$ in the limit $\left|K \operatorname{Im}\left(f_{l}\right)\right| \rightarrow \Delta$ these eigenvalues approach the continuous spectrum and are "absorbed" as the underlying root $z_{u}$ or $z_{s}$ crosses the branch cut onto a different Riemann sheet of $\Lambda_{l}(z)$. 
The diffusion due to external noise simplifies the spectrum by displacing the lines of continuous spectrum into the left half plane. With $D>0$ the eigenvalues associated with instabilities first emerge from the continuum and then subsequently reach the imaginary axis. Thus, the critical eigenvalues are isolated on the imaginary axis, and one has a critical spectrum of standard form for bifurcation theory. If we let $D \rightarrow 0$, then the unstable eigenvalues emerge directly from a continuous spectrum on the imaginary axis. One of our central concerns is the effect of this continuous spectrum for $D=0$ on the $\gamma \rightarrow 0^{+}$limit of the vector field on the center-unstable manifold. This is investigated below by deriving this vector field first for $D>0$ and then analyzing the effect of taking the limit $\gamma \rightarrow 0^{+}$when $D=0$.

\section{Amplitude equation on the unstable manifold}

With diffusion included, the appearance of an unstable mode in the spectrum can be analyzed by a straightforward application of center manfold reduction. [20, 21] This enables us to derive approximately the vector field on the center-unstable manifold of $\rho_{0}$, and study the resulting bifurcation. This vector field will depend on the parameters of the problem and we shall examine its features as the growth rate of the unstable mode is progressively weakened, a limit which we denote by $\gamma \rightarrow 0^{+}$. The properties of this limit are strongly dependent on the form of the coupling $f(\phi)$ and whether the external noise $D>0$ is held fixed as $\gamma$ vanishes.

Center manifold reduction yields an amplitude equation describing the time-asymptotic behavior of the unstable mode. The mode amplitude $\alpha(t) \equiv(\tilde{\Psi}, \eta)$ and describes the projection of $\eta$ onto the unstable eigenspace (30):

$$
\eta(\theta, \omega, t)=[\alpha(t) \Psi(\theta, \omega)+\text { c.c. }]+S(\theta, \omega, t)
$$


where $(\tilde{\Psi}, S)=0$. In terms of $\alpha$ and $S$, the evolution equation (10) becomes

$$
\begin{aligned}
\dot{\alpha} & =\lambda \alpha+(\tilde{\Psi}, \mathcal{N}(\eta)) \\
\frac{\partial S}{\partial t} & =\mathcal{L} S+\mathcal{N}(\eta)-[(\tilde{\Psi}, \mathcal{N}(\eta)) \Psi+\text { c.c. }]
\end{aligned}
$$

These coupled equations are equivalent to $(10)$; by restricting them to solutions on the center-unstable manifold we obtain an autonomous two-dimensional flow for $\alpha(t)$.

Near the equilibrium $\eta=0$, the center-unstable manifold is described by a function $H$,

$$
S(\theta, \omega)=H\left(\theta, \omega, \alpha, \alpha^{*}\right)
$$

that satisfies $0=H(\theta, \omega, 0,0)=\partial_{\alpha} H(\theta, \omega, 0,0)=\partial_{\alpha^{*}} H(\theta, \omega, 0,0)$. Using this representation of the manifold, the general solution in (43) can be adapted for solutions on the manifold,

$$
\eta^{u}(\theta, \omega, t)=[\alpha(t) \Psi(\theta, \omega)+\text { c.c. }]+H\left(\theta, \omega, \alpha(t), \alpha(t)^{*}\right)
$$

the time dependence of $\eta^{u}(\theta, \omega, t)$ is entirely determined by the dynamics of $\alpha(t)$. An autonomous equation for $\alpha(t)$ follows next by restricting the general equation (44) to solutions of the special form (47):

$$
\dot{\alpha}=\lambda \alpha+\left(\tilde{\Psi}, \mathcal{N}\left(\eta^{u}\right)\right)
$$

This is the amplitude equation we wish to derive and analyze.

Certain general features of the amplitude equation follow from the symmetry properties of the evolution equation (10) which are, of course, shared by its expression in (44) - (45). The action of the rotations $\beta$ and reflection $\kappa$ on the variables $(\alpha, S(\theta, \omega))$ can be determined from their definitions in (43). The rotation $\beta \cdot(\theta, \omega) \rightarrow(\theta+\beta, \omega)$ of the phase acts by

$$
\beta \cdot(\alpha, S(\theta, \omega))=\left(\alpha e^{-i l \beta}, S(\theta-\beta, \omega)\right)
$$

and

$$
\kappa \cdot(\alpha, S(\theta, \omega))=\left(\alpha^{*}, S(-\theta,-\omega)\right)
$$


for the reflection.

The right hand side of the amplitude equation commutes with these transformations of $\alpha$ whenever they are symmetries of (44) - (45); in particular the amplitude equation commutes with rotations (49). There are useful standard results on the expression of such equivariant vector fields. For example, a two-dimensional vector field $\dot{\alpha}=V\left(\alpha, \alpha^{*}\right)$ that commutes with $\alpha \rightarrow \alpha e^{-i l \beta}$ can be written as $V\left(\alpha, \alpha^{*}\right)=\alpha p(\sigma)$ where $\sigma \equiv|\alpha|^{2}$ and $p(\sigma)$ is a smooth function. 33 Hence we know the amplitude equation (48) has the form

$$
\lambda \alpha+\left(\tilde{\Psi}, \mathcal{N}\left(\eta^{u}\right)\right)=\alpha p(\sigma)
$$

although $p(\sigma)$ must be determined from the model. In general, $p(\sigma)$ is complex, but when the model has reflection symmetry then $p(\sigma)$ must be real-valued.

\subsection{Analysis of $H\left(\theta, \omega, \alpha, \alpha^{*}\right)$}

The dynamical invariance of the center-unstable manifold implies an equation for the graph function $H$. Consistency between the time dependence of $S$ in (46) and the evolution of $S$ described by (44)-(45) requires that $H\left(\theta, \omega, \alpha(t), \alpha^{*}(t)\right)$ satisfy

$$
\left[\dot{\alpha} \frac{\partial H}{\partial \alpha}+\dot{\alpha}^{*} \frac{\partial H}{\partial \alpha^{*}}\right]=\mathcal{L} H+\mathcal{N}\left(\eta^{u}\right)-\left[\left(\tilde{\Psi}, \mathcal{N}\left(\eta^{u}\right)\right) \Psi+\left(\tilde{\Psi}, \mathcal{N}\left(\eta^{u}\right)\right)^{*} \Psi^{*}\right]
$$

where $\dot{\alpha}$ on the left hand side refers to the amplitude equation (48).

There are general constraints on $H$ due to the symmetries of (44) - (45) because we expect the unstable manifold to be mapped onto itself by a symmetry transformation. For a rotation $\beta$, this symmetry invariance of the manifold implies

$$
H\left(\theta-\beta, \omega, \alpha, \alpha^{*}\right)=H\left(\theta, \omega, \alpha e^{-i l \beta}, \alpha^{*} e^{i l \beta}\right),
$$

and if reflection symmetry holds then $H$ must satisfy

$$
H\left(-\theta,-\omega, \alpha, \alpha^{*}\right)=H\left(\theta, \omega, \alpha^{*}, \alpha\right)
$$


as well.

These relations constrain the form of the Fourier expansion of $\mathrm{H}$

$$
H\left(\theta, \omega, \alpha, \alpha^{*}\right)=\sum_{n=-\infty}^{\infty} H_{n}\left(\omega, \alpha, \alpha^{*}\right) e^{i n \theta} ;
$$

applying (53) to the components $H_{n}$ shows that the component must vanish unless $n$ is an integer multiple of $l$. Note that the $n=0$ component of $H$ is also identically zero because of the general property $\int d \theta \eta=0$. When $n=m l>0$, then $H_{m l}$ must have the form

$$
\begin{aligned}
H_{l}\left(\omega, \alpha, \alpha^{*}\right) & =\alpha \sigma h_{1}(\omega, \sigma) \\
H_{m l}\left(\omega, \alpha, \alpha^{*}\right) & =\alpha^{m} h_{m}(\omega, \sigma) \quad m>1 .
\end{aligned}
$$

The functions $h_{m}(\omega, \sigma)$ are unconstrained by the rotations, but must satisfy $h_{m}(-\omega, \sigma)^{*}=$ $h_{m}(\omega, \sigma)$ when reflection symmetry holds. In addition, the general relation $(\tilde{\Psi}, S)=0$ becomes $(\tilde{\Psi}, H)=0$ for solutions on the unstable manifold, and for the components of $H$ this implies

$$
<\tilde{\psi}, h_{1}>=0
$$

The symmetry argument for (56) and (57) can be briefly summarized. Applying (53) to the Fourier coefficients of $H$ leads to the identity

$$
H_{n}\left(\omega, \alpha, \alpha^{*}\right) e^{-i n \beta}=H_{n}\left(\omega, \alpha e^{-i l \beta}, \alpha^{*} e^{i l \beta}\right)
$$

For $\beta=2 \pi / l$ this immediately implies that $H_{n}=0$ unless $\exp (-i 2 \pi n / l)=1$, hence $n$ must be an integer multiple of $l$. Recall that if a function $F\left(\omega, \alpha, \alpha^{*}\right)$ is rotation invariant under phase shifts $\alpha \rightarrow \alpha \exp -i l \beta$, then it can be written as $F\left(\omega, \alpha, \alpha^{*}\right)=g(\omega, \sigma)$ where the form of $g(\omega, \sigma)$ is determined by $F$. 33 In this case, the invariant function $\left(\alpha^{*}\right)^{m} H_{m l}\left(\omega, \alpha, \alpha^{*}\right)=$ $g(\omega, \sigma)$ must have the form $g(\omega, \sigma)=\sigma^{m} h(\omega, \sigma)$ to accomodate the factor $\left(\alpha^{*}\right)^{m}$. Dividing by $\left(\alpha^{*}\right)^{m}$ gives $H_{m l}\left(\omega, \alpha, \alpha^{*}\right)=\alpha^{m} h(\omega, \sigma)$ as in (57). The general property $\partial_{\alpha} H(\theta, \omega, 0,0)=0$ requires that $h(\omega, \sigma)$ for $H_{l}$ have the special form $h(\omega, \sigma)=\sigma h_{1}(\omega, \sigma)$ as in (56). 
The equations for $h_{m}(\omega, \sigma)$ follow by substituting (56) - (57) and (51) into the equation for $H$ (52). The results of this straightforward calculation for $m=1,2$ and $m>2$ are summarized below. In writing these equations two further notations prove convenient. First, define $\Gamma_{m}$ by

$$
\Gamma_{m}(\sigma) \equiv \int_{-\infty}^{\infty} d \omega g(\omega) h_{m}(\omega, \sigma),
$$

and secondly let $\mathrm{P}_{\perp}$ denote the projection operator

$$
\left(\mathrm{P}_{\perp} \phi\right)(\omega) \equiv \phi(\omega)-<\tilde{\psi}, \phi>\psi(\omega)
$$

which projects $\phi$ onto the orthogonal complement of the eigenvector $\psi$.

The $n=l$ component $(m=1)$ of $(52)$ can be written

$$
\begin{aligned}
& {\left[L_{l}-2 p-p^{*}\right] h_{1}-\left(p+p^{*}\right) \sigma \frac{\partial h_{1}}{\partial \sigma}=(2 \pi i K l) \mathrm{P}_{\perp} }\left\{f_{l}\left(1+\sigma \Gamma_{1}^{*}\right) h_{2}+f_{2 l}^{*} \Gamma_{2}\left(\psi^{*}+\sigma h_{1}^{*}\right)\right. \\
&+ f_{2 l} \sigma \Gamma_{2}^{*} h_{3}+f_{3 l}^{*} \sigma \Gamma_{3} h_{2}^{*} \\
&\left.+\sum_{m=3}^{\infty} \sigma^{m-1}\left[f_{m l} \Gamma_{m}^{*} h_{m+1}+f_{(m+1) l}^{*} \Gamma_{m+1} h_{m}^{*}\right]\right\} .
\end{aligned}
$$

The $n=2 l$ component of (52) is

$$
\begin{aligned}
{\left[L_{2 l}-2 p\right] h_{2}-\left(p+p^{*}\right) \sigma \frac{\partial h_{2}}{\partial \sigma}=(} & \pi i K(2 l))\left\{f_{l}^{*}\left[\psi+\sigma\left(h_{1}+\Gamma_{1} \psi\right)+\sigma^{2} \Gamma_{1} h_{1}\right]\right. \\
& +f_{l} \sigma\left(1+\sigma \Gamma_{1}^{*}\right) h_{3}+f_{3 l}^{*} \sigma\left[\Gamma_{3} \psi^{*}+\sigma \Gamma_{3} h_{1}^{*}\right] \\
& \left.+\sum_{m=2}^{\infty} \sigma^{m} f_{m l} \Gamma_{m}^{*} h_{m+2}+\sum_{m=4}^{\infty} \sigma^{m-2} f_{m l}^{*} \Gamma_{m} h_{m-2}^{*}\right\} .
\end{aligned}
$$

For $m \geq 3$, the Fourier components of (52) are

$$
\begin{aligned}
{\left[L_{m l}-m p\right] h_{m}-\left(p+p^{*}\right) \sigma \frac{\partial h_{m}}{\partial \sigma}=(} & \pi i K(m l))\left\{f_{l}^{*}\left[h_{m-1}+\sigma \Gamma_{1} h_{m-1}\right]+f_{l} \sigma\left(1+\sigma \Gamma_{1}^{*}\right) h_{m+1}\right. \\
& +\sum_{n=2}^{m-2} f_{(m-n) l}^{*} \Gamma_{m-n} h_{n} \\
& +f_{(m-1) l}^{*} \Gamma_{m-1}\left[\psi+\sigma h_{1}\right]+f_{(m+1) l}^{*} \sigma \Gamma_{m+1}\left[\psi^{*}+\sigma h_{1}^{*}\right] \\
& \left.+\sum_{n=2}^{\infty} f_{n l} \sigma^{n} \Gamma_{n}^{*} h_{m+n}+\sum_{n=2}^{\infty} f_{(m+n) l}^{*} \sigma^{n} \Gamma_{m+n} h_{n}^{*}\right\} .
\end{aligned}
$$


Here and below, it is understood that sums are omitted when the lower value of the summation index exceeds the upper value, e.g. for $m=3$ the first sum in (64) is omitted.

The symmetry constraints imply that solutions on the unstable manifold (47) have the general form

$$
\begin{aligned}
\eta^{u}(\theta, \omega, t)=[\alpha(t) \Psi(\theta, \omega) & +\alpha(t) \sigma h_{1}(\omega, \sigma(t)) e^{i l \theta} \\
& \left.+\sum_{m=2}^{\infty} \alpha^{m}(t) h_{m}(\omega, \sigma(t)) e^{i m l \theta}\right]+ \text { c.c. }
\end{aligned}
$$

with $\alpha(t)$ satisfying the amplitude equation (48). The set of equations (51) and (62) - (64) determine the functions $p(\sigma)$ and $h_{m}(\omega, \sigma)$, however they cannot be solved explicitly except using the amplitude expansions introduced in the next section. What has been achieved to this point is to formulate the problem entirely in terms of functions of a single real variable $\sigma$. This simplification is essential to the analysis to follow.

\section{Expansions and recursion relations}

Our conclusions regarding the nonlinear development of the instability are based on amplitude expansions for $p$ and $h_{m}(\omega, \sigma)$, and the corresponding expansion of $\Gamma_{m}$ from (60):

$$
\begin{aligned}
p(\sigma) & =\sum_{j=0}^{\infty} p_{j} \sigma^{j} \\
h_{m}(\omega, \sigma) & =\sum_{j=0}^{\infty} h_{m, j}(\omega) \sigma^{j} \\
\Gamma_{m}(\sigma) & =<g, h_{m}>=\sum_{j=0}^{\infty} \Gamma_{m, j} \sigma^{j}
\end{aligned}
$$

where $\Gamma_{m, j} \equiv \int d \omega g h_{m, j}$. The orthogonality condition (58) implies

$$
<\tilde{\psi}, h_{1, j}>=0
$$

at each order in the expansion of $h_{1}$. 
The coefficients $p_{j}$ and $h_{m, j}(\omega)$ are determined by recursively solving (51) and (62) - (64). Relations for $p_{j}$ follow from (51) using the general solution form in (65) and the expansions (66) - (68). At each order in $\sigma^{j}, j \geq 1$, we solve (51) for $p_{j}$ and find

$$
\begin{aligned}
p_{j}=-( & 2 \pi i K l)\left\{f_{l}\left[<\tilde{\psi}, h_{2, j-1}>+\sum_{n=0}^{j-2} \Gamma_{1, n}^{*}<\tilde{\psi}, h_{2, j-n-2}>\right]\right. \\
& +f_{2 l}^{*}\left[\Gamma_{2, j-1}<\tilde{\psi}, \psi^{*}>+\sum_{n=0}^{j-2} \Gamma_{2, n}<\tilde{\psi}, h_{1, j-n-2}^{*}>\right]+f_{2 l} \sum_{n=0}^{j-2} \Gamma_{2, n}^{*}<\tilde{\psi}, h_{3, j-n-2}> \\
& \left.+\sum_{m=3}^{j+1} f_{m l}^{*} \sum_{n=0}^{j-m+1} \Gamma_{m, n}<\tilde{\psi}, h_{m-1, j-n-m+1}^{*}>+\sum_{m=3}^{j} f_{m l} \sum_{n=0}^{j-m} \Gamma_{m, n}^{*}<\tilde{\psi}, h_{m+1, j-n-m}>\right\} .
\end{aligned}
$$

The functions $h_{m, j}(\omega)$ are determined similarly by substituting (66) - (68) into the component equations (62) - (64). At every order, $h_{m, j}$ is then obtained by applying the resolvent operator (17) to certain auxilliary functions $I_{m, j}(\omega)$ constructed from lower order terms, i.e.

$$
h_{m, j}=-R_{m l}\left(\mu_{m, j}\right) I_{m, j}(\omega)
$$

where

$$
\mu_{m, j} \equiv(m+j) \lambda+j \lambda^{*}+\delta_{m, 1}\left(\lambda+\lambda^{*}\right),
$$

and the detailed expressions for $I_{m, j}$ are provided below.

The action of the resolvent is given in (17)

$R_{m l}\left(\mu_{m, j}\right) I_{m, j}=\frac{-i / m l}{\omega+K f_{0}-\nu_{m, j}-i m l D}\left[I_{m, j}(\omega)-\frac{K f_{m l}^{*}}{\Lambda_{m l}\left(\nu_{m, j}\right)} \int_{-\infty}^{\infty} \frac{d \omega^{\prime} g\left(\omega^{\prime}\right) I_{m, j}\left(\omega^{\prime}\right)}{\omega^{\prime}+K f_{0}-\nu_{m, j}-i m l D}\right]$

with $\nu_{m, j} \equiv i \mu_{m, j} / m l$. It is important to note from (72) that $\nu_{m, j}$ can be rewritten

$$
\nu_{m, j}=z_{0}+\frac{2 i\left(j+\delta_{m, 1}\right) \gamma}{m l}
$$

hence for $m \geq 1$, the poles $\omega=\nu_{m, j}-K f_{0}+i m l D$ in (73) all sit in the upper half of the complex $\omega$ plane along the line $\operatorname{Re}(\omega)=\operatorname{Re}\left(z_{0}\right)-K f_{0}$. 
The coefficients $p_{j}$ are determined by the integrals $\Gamma_{m, j},\left\langle\tilde{\psi}, h_{m, j}\right\rangle,\left\langle\tilde{\psi}, h_{m, j}^{*}\right\rangle$, and $<\tilde{\psi}, \psi^{*}>$. As we are interested in the form of these coefficients near the onset of the instability $(\gamma \rightarrow 0)$ when the noise is neglected (i.e. $D=0$ ), it is important to determine the behavior of such integrals in this limit. In particular, we will need to estimate any singular behavior exhibited by each type of integral.

In the remainder of the paper, our analysis of the amplitude expansion will be facilitated by two identities. From the definition of $\Gamma_{m, j}$ in (68) and the resolvent expression for $h_{m, j}(\omega)$ in (71), the following useful relations are obtained

$$
\begin{aligned}
\Gamma_{m, j} & =\frac{i / m l}{\Lambda_{m l}\left(\nu_{m, j}\right)} \int_{-\infty}^{\infty} d \omega \frac{g(\omega) I_{m, j}(\omega)}{\left(\omega+K f_{0}-\nu_{m, j}-i m l D\right)} \\
h_{m, j}(\omega) & =\frac{i I_{m, j}(\omega)}{m l\left(\omega+K f_{0}-\nu_{m, j}-i m l D\right)}-\frac{K f_{m l}^{*} \Gamma_{m, j}}{\left(\omega+K f_{0}-\nu_{m, j}-i m l D\right)} .
\end{aligned}
$$

Given $I_{m, j}$, these identities allow the properties of $h_{m, j}$ and $\Gamma_{m, j}$ to be quickly determined.

The $I_{m, j}$ are in turn calculated from the following recursion relations in terms of lower order coefficients. The application of these recursion relations to calculate the coefficients $p_{j}$ is summarized in Table 1 and illustrated in Section 5 .

\subsection{Recursion relations for $I_{m, j}$}

The expansion of (62) using (66) - (68) determines $h_{1, j}$ at each order in (73): $h_{1, j}=$ $-R_{l}\left(\mu_{1, j}\right) I_{1, j}$ where for $m=1$ and $j \geq 0$,

$$
\begin{aligned}
& I_{1, j}=\sum_{k=0}^{j-1}\left[(2+k) p_{j-k}+(1\right.\left.+k) p_{j-k}^{*}\right] h_{1, k} \\
&+(2 \pi i K l) \mathrm{P}_{\perp}\left\{f_{l}\left[h_{2, j}+\sum_{n=0}^{j-1} h_{2, n} \Gamma_{1, j-n-1}^{*}\right]\right. \\
&+f_{2 l}^{*}\left[\psi^{*} \Gamma_{2, j}+\sum_{n=0}^{j-1} h_{1, n}^{*} \Gamma_{2, j-n-1}\right]+f_{2 l} \sum_{n=0}^{j-1} h_{3, n} \Gamma_{2, j-n-1}^{*}
\end{aligned}
$$




$$
\left.+\sum_{m=3}^{j+2} f_{m l}^{*} \sum_{n=0}^{j-m+2} h_{m-1, n}^{*} \Gamma_{m, j-m-n+2}+\sum_{m=3}^{j+1} f_{m l} \sum_{n=0}^{j-m+1} h_{m+1, n} \Gamma_{m, j-m-n+1}^{*}\right\} .
$$

Since $<\tilde{\psi}, \mathrm{P}_{\perp} \phi>=0$ by construction, this recursion relation together with the orthogonality property of $h_{1, j}$ (69) implies a corresponding orthogonality property for $I_{1, j}$

$$
<\tilde{\psi}, I_{1, j}>=0 \text {. }
$$

For $m=2$, the expansion of (63) yields $h_{2, j}=-R_{l}\left(\mu_{2, j}\right) I_{2, j}$ where for $j=0$

$$
I_{2,0}=2 \pi i K(2 l) f_{l}^{*} \psi(\omega)
$$

and for $j \geq 1$,

$$
\begin{array}{rl}
I_{2, j}=\sum_{k=0}^{j-1}\left[2 p_{j-k}+k\left(p_{j-k}+p_{j-k}^{*}\right)\right] h_{2, k} \\
+2 & i k(2 l)\left\{f_{l}\left[h_{3, j-1}+\sum_{n=0}^{j-2} h_{3, n} \Gamma_{1, j-n-2}^{*}\right]\right. \\
+ & f_{l}^{*}\left[h_{1, j-1}+\Gamma_{1, j-1} \psi+\sum_{n=0}^{j-2} h_{1, n} \Gamma_{1, j-n-2}\right]+f_{2 l} \sum_{n=0}^{j-2} h_{4, n} \Gamma_{2, j-n-2}^{*} \\
+ & f_{3 l}^{*}\left[\psi^{*} \Gamma_{3, j-1}+\sum_{n=0}^{j-2} h_{1, n}^{*} \Gamma_{3, j-n-2}\right]+f_{3 l} \sum_{n=0}^{j-3} h_{5, n} \Gamma_{3, j-n-3}^{*} \\
& \left.+\sum_{k=4}^{j+2} f_{k l}^{*} \sum_{n=0}^{j-k+2} h_{k-2, n}^{*} \Gamma_{k, j-n-k+2}+\sum_{k=4}^{j} f_{k l} \sum_{n=0}^{j-k} h_{k+2, n} \Gamma_{k, j-n-k}^{*}\right\}
\end{array}
$$

For $m \geq 3$ and $j \geq 0$ we have $h_{m, j}=-R_{m l}\left(\mu_{m, j}\right) I_{m, j}$ with

$$
\begin{aligned}
& I_{m, j}=\sum_{k=0}^{j-1}\left[m p_{j-k}+k\left(p_{j-k}+p_{j-k}^{*}\right)\right] h_{m, k}(81) \\
&+2 \pi i K(m l)\left\{f_{l}\left[h_{m+1, j-1}+\sum_{k=0}^{j-2} h_{m+1, k} \Gamma_{1, j-k-2}^{*}\right]+f_{l}^{*}\left[h_{m-1, j}+\sum_{k=0}^{j-1} h_{m-1, k} \Gamma_{1, j-k-1}\right]\right. \\
& \quad+f_{(m-1) l}^{*}\left[\psi \Gamma_{m-1, j}+\sum_{k=0}^{j-1} h_{1, k} \Gamma_{m-1, j-k-1}\right]+\sum_{n=2}^{m-2} f_{n l}^{*} \sum_{k=0}^{j} h_{m-n, k} \Gamma_{n, j-k}
\end{aligned}
$$




$$
\left.\begin{array}{rl}
+f_{(m+1) l}^{*} & {\left[\psi^{*} \Gamma_{m+1, j-1}+\sum_{k=0}^{j-2} h_{1, k}^{*} \Gamma_{m+1, j-k-2}\right]} \\
+ & \sum_{n=2}^{j} f_{n l} \sum_{k=0}^{j-n} h_{m+n, k} \Gamma_{n, j-n-k}^{*}+\sum_{n=m+2}^{m+j} f_{n l}^{*} \sum_{k=0}^{j-n+m} h_{n-m, k}^{*} \Gamma_{n, j-n-k+m}
\end{array}\right\} .
$$

In this final expression it is understood that a term with a negative subscript is omitted, e.g. the term $f_{l} h_{m+1, j-1}$ is dropped when $j=0$.

\section{Pinching singularities at low order}

At this point it is instructive to evaluate the low order coefficients in the expansion and understand how the singularities arise mathematically. We first calculate the cubic coefficient $p_{1}$ and show that it has a singularity when $f_{2 l} \neq 0$. Then we evaluate the fifth order coefficient for the special case of a coupling with $f_{2 l}=0$ and no singularity in $p_{1}$.

\subsection{Evaluation of the coefficient $p_{1}$}

The calculation of $p_{1}$ in (170) yields

$$
p_{1}=-2 \pi i K l\left[f_{l}<\tilde{\psi}, h_{2,0}>+f_{2 l}^{*} \Gamma_{2,0}<\tilde{\psi}, \psi^{*}>\right]
$$

where the function $h_{2,0}$ is determined from (76) and (79)

$$
h_{2,0}(\omega)=\frac{i I_{2,0}}{2 l\left(\omega+K f_{0}-z_{0}-i 2 l D\right)}-\frac{K f_{2 l}^{*} \Gamma_{2,0}}{\left(\omega+K f_{0}-z_{0}-i 2 l D\right)}
$$

with $\Gamma_{2,0}$ given by $(75)$

$$
\Gamma_{2,0}=\frac{i / 2 l}{\Lambda_{2 l}\left(z_{0}\right)} \int d \omega \frac{g(\omega) I_{2,0}(\omega)}{\left(\omega+K f_{0}-z_{0}-i 2 l D\right)}=\frac{2 \pi K f_{l}^{*} \Lambda_{l}^{\prime}\left(z_{0}\right)}{\Lambda_{2 l}\left(z_{0}\right)}+\mathcal{O}(D) .
$$

The asymptotic expression for small $D$ in (84) shows that generically $\Gamma_{2,0} \neq 0$.

The most important qualitative feature of $I_{2,0}(\omega)$ and $h_{2,0}(\omega)$ is that they are meromorphic functions on the complex $\omega$ plane. Let $z_{0}=(\Omega+i \gamma) / l$ denote the real and imaginary 
parts of the root, then each function has a pole at $\omega_{+}=\left(\Omega / l-K f_{0}\right)+i\left(l^{2} D+\gamma\right) / l$. In addition, $h_{2,0}(\omega)$ has a second pole at $\left(\Omega / l-K f_{0}\right)+i\left(2 l^{2} D+\gamma\right) / l$. Both poles lie in the upper half plane and although they can approach the real axis as the $\gamma \rightarrow 0^{+}$, the integrals $<\tilde{\psi}, h_{2,0}>$ and $\Gamma_{2,0}$ in (82) have well-behaved nonsingular limits even if $D$ is zero.

This observation regarding analytic structure can be extended considerably: all of the functions $I_{m, j}(\omega)$ and $h_{m, j}(\omega)$ are meromorphic functions in the complex $\omega$ plane and all of their poles lie along the vertical line $\operatorname{Re}(\omega)=-K f_{0}+\operatorname{Re}\left(z_{0}\right)$. When $D=0$, all of the poles approach the real axis in the limit $\gamma \rightarrow 0^{+}$; in general, however the poles will be found in both the upper and lower half plane and integrals over $I_{m, j}(\omega)$ or $h_{m, j}(\omega)$ will exhibit pinching singularities. These conclusions follow from the recursion relations in light of two simple observations. First, each $I_{m, j}(\omega)$ is built up as a sum of various $h_{m, j}(\omega)$ from lower order and the sum of two meromorphic functions is again meromorphic. Secondly, the application of the resolvent (73) to a meromorphic $I_{m, j}(\omega)$ yields a meromorphic function for $h_{m, j}(\omega)$ in (76).

The simplest pinching singularity is illustrated by $<\tilde{\psi}, \psi^{*}>$ in $p_{1}$; from (30) and (37) one sees that the integrand has poles $\omega_{ \pm}=\left(\Omega / l-K f_{0}\right) \pm i\left(l^{2} D+\gamma\right) / l$ above and below the contour along the real axis. Now the $\gamma \rightarrow 0^{+}$limit produces a pinching singularity when $D$ is zero, and this pinch contributes a factor $\left(\gamma+l^{2} D\right)$ to the denominator when the integral is evaluated:

$$
<\tilde{\psi}, \psi^{*}>=-\frac{l f_{l} \operatorname{Im}\left(f_{l}\right)}{\left(\gamma+l^{2} D\right)\left|f_{l}\right|^{2} \Lambda_{l}^{\prime}\left(z_{0}\right)} .
$$

This singularity will occur in the $p_{1}$ coefficient (82) provided $D \approx 0$ and $f_{2 l} \neq 0$.

The derivation of (85) is simple and illustrates the key idea used below in the general analysis: make a partial fraction expansion of the integrand to isolate the singularity. In 
this case we have

$$
<\tilde{\psi}, \psi^{*}>=\frac{K f_{l}}{\Lambda_{l}^{\prime}\left(z_{0}\right)} \int_{-\infty}^{\infty} d \omega \frac{g(\omega)}{\left(\omega-\omega_{+}\right)\left(\omega-\omega_{-}\right)} .
$$

The partial fraction expansion yields

$$
\frac{1}{\left(\omega-\omega_{+}\right)\left(\omega-\omega_{-}\right)}=\frac{-i l / 2}{\gamma+l^{2} D}\left(\frac{1}{\left(\omega-\omega_{+}\right)}-\frac{1}{\left(\omega-\omega_{-}\right)}\right),
$$

so that (86) becomes

$$
<\tilde{\psi}, \psi^{*}>=\frac{-i l K f_{l}}{2 \Lambda_{l}^{\prime}\left(z_{0}\right)\left(\gamma+l^{2} D\right)}\left(\frac{\Lambda_{l}\left(z_{0}\right)-1}{K f_{l}^{*}}-\frac{\Lambda_{l}\left(z_{0}\right)^{*}-1}{K f_{l}}\right) .
$$

This reduces to (85) since $\Lambda_{l}\left(z_{0}\right)=0$. For $D=0$, the cubic coefficient diverges like $p_{1} \sim \gamma^{-1}$ as $\gamma \rightarrow 0^{+}$provided $f_{2 l} \neq 0$; this turns out to reflect a general pattern $p_{j} \sim \gamma^{-2 j+1}$ which is proved below in Theorem 6.1.

The significance of this divergence can be appreciated by considering the amplitude equation truncated at third order

$$
\dot{\alpha}=\lambda \alpha+p_{1} \alpha|\alpha|^{2}
$$

and re-examining the determination of $\beta$ in (6). Introducing polar variables $\alpha=A(t) \exp (-i \psi(t))$, the amplitude equation reads

$$
\dot{A}=\gamma A+\operatorname{Re}\left(p_{1}\right) A^{3} \quad \dot{\psi}=\Omega-\operatorname{Im}\left(p_{1}\right) A^{2}
$$

where $\lambda=\gamma-i \Omega$. Let $p_{1}=a_{1} \gamma^{-\nu}$ denote the asymptotic behavior of the cubic coefficient with $\nu \geq 0$, then in terms of the rescaled amplitude (7) the equations become

$$
\frac{d r}{d \tau}=\gamma^{1-\delta} r+\gamma^{2 \beta-\nu-\delta} \operatorname{Re}\left(a_{1}\right) r^{3} \quad \dot{\psi}=\Omega-\gamma^{2 \beta-\nu} \operatorname{Im}\left(a_{1}\right) r^{2} .
$$

In order to remove the singular perturbation due to the linear term we again take $\delta=1$ and then solve for $\beta=(1+\nu) / 2$ to balance the cubic nonlinearity against the linear instability. This makes it clear that a divergence in the cubic coefficient will increase $\beta$ and for $\nu=1$ we 
predict $\beta=1$. This of course neglects possible effects from higher order nonlinear terms, in particular singularities at higher order could turn out to be more important than the cubic singularity. The effects of higher order singularities are systematically studied in section 6 .

\subsection{The fifth order coefficient $p_{2}$}

When $f_{2 l}=0$ the cubic coefficient is nonsingular, and we briefly consider what occurs at fifth order in the amplitude expansion. Setting $f_{2 l}=0$, the fifth order coefficient is given by

$$
p_{2}=-(2 \pi i K l)\left\{f_{l}\left[<\tilde{\psi}, h_{2,1}>+\Gamma_{1,0}^{*}<\tilde{\psi}, h_{2,0}>\right]+f_{3 l}^{*} \Gamma_{3,0}<\tilde{\psi}, h_{2,0}^{*}>\right\} \text {. }
$$

where

$$
\begin{aligned}
I_{1,0}(\omega)= & (2 \pi i K l) \mathrm{P}_{\perp}\left\{f_{l} h_{2,0}\right\}=(2 \pi i K l) f_{l}\left\{h_{2,0}(\omega)-\psi(\omega)<\tilde{\psi}, h_{2,0}>\right\} \\
I_{3,0}(\omega)=2 \pi i K(3 l) f_{l}^{*} h_{2,0}(\omega) & \\
I_{2,1}(\omega)=2 p_{1} h_{2,0}(\omega)+2 \pi i K(2 l)\left[f_{l} h_{3,0}(\omega)+\right. & f_{l}^{*}\left(h_{1,0}(\omega)+\Gamma_{1,0} \psi(\omega)\right) \\
& \left.+f_{3 l}^{*} \Gamma_{3,0} \psi^{*}(\omega)\right] .
\end{aligned}
$$

There are five integrals to consider in (92). Since $h_{2,0}$ has poles only in the upper plane,

the integral $<\tilde{\psi}, h_{2,0}>$ is not singular. From (94), we note that $I_{3,0}$ similarly has poles only in the upper half plane hence $\Gamma_{3,0}$ has no pinching singularity. In addition, $I_{1,0}$ in (93) has poles in the upper plane only and this implies that $\Gamma_{1,0}$ is nonsingular, but the calculation is more subtle. From (75) we have

$$
\Gamma_{1,0}=\frac{i / l}{\Lambda_{l}\left(\nu_{1,0}\right)} \int_{-\infty}^{\infty} d \omega \frac{g(\omega) I_{1,0}(\omega)}{\left(\omega+K f_{0}-\nu_{1,0}-i l D\right)} .
$$

Since $\Lambda_{l}\left(\nu_{1,0}\right)=\Lambda_{l}\left(z_{0}+2 \gamma / l\right)$ from Eq. (74), the first factor in (96) is singular as $\gamma \rightarrow 0^{+}$. However the integral is nonsingular in this limit and because of the orthogonality relation (88) the leading term is zero so the integral is actually $\mathcal{O}(\gamma)$. The vanishing of the integral as $\gamma \rightarrow 0^{+}$cancels the singularity from $\Lambda_{l}\left(\nu_{1,0}\right)$ and $\Gamma_{1,0}$ is well-behaved. 
The remaining integrals do exhibit pinching singularities which can be evaluated with the partial fraction procedure illustrated above. Rewriting $p_{2}$ to isolate these singular terms we find for $D=0$ :

$$
p_{2}=-(2 \pi i K l)\left\{f_{l}<\tilde{\psi}, h_{2,1}>+f_{3 l}^{*} \Gamma_{3,0}<\tilde{\psi}, h_{2,0}^{*}>+ \text { nonsingular terms }\right\}
$$

with

$$
\begin{aligned}
& <\tilde{\psi}, h_{2,1}>=-2 \pi K f_{3 l}^{*} \Gamma_{3,0} \int_{-\infty}^{\infty} \frac{d \omega \tilde{\psi}(\omega)^{*} \psi(\omega)^{*}}{\omega+K f_{0}-\left(z_{0}+i \gamma / l\right)}+\text { nonsingular terms } \\
& <\tilde{\psi}, h_{2,0}^{*}>=-2 \pi K f_{l} \int_{-\infty}^{\infty} \frac{d \omega \tilde{\psi}(\omega)^{*} \psi(\omega)^{*}}{\omega+K f_{0}-z_{0}^{*}}
\end{aligned}
$$

A partial fraction expansion of each of these integrals allows an explicit evaluation of the singular behavior

$$
\begin{aligned}
\int_{-\infty}^{\infty} \frac{d \omega \tilde{\psi}(\omega)^{*} \psi(\omega)^{*}}{\omega+K f_{0}-\left(z_{0}+i \gamma / l\right)} & =-\frac{i l^{2} f_{l}\left[\operatorname{Im}\left(f_{l}\right)+\mathcal{O}(\gamma)\right]}{3\left|f_{l}\right|^{2} \Lambda_{l}^{\prime}\left(z_{0}\right) \gamma^{2}} \\
\int_{-\infty}^{\infty} \frac{d \omega \tilde{\psi}(\omega)^{*} \psi(\omega)^{*}}{\omega+K f_{0}-z_{0}^{*}} & =\frac{i l^{2} f_{l}\left[\operatorname{Im}\left(f_{l}\right)+\mathcal{O}(\gamma)\right]}{2\left|f_{l}\right|^{2} \Lambda_{l}^{\prime}\left(z_{0}\right) \gamma^{2}} ;
\end{aligned}
$$

each integral diverges like $\gamma^{-2}$.

The resulting evaluation of $p_{2}$ yields

$$
p_{2}=-\frac{2 l\left(\pi l K f_{l}\right)^{2} f_{3 l}^{*} \Gamma_{3,0}\left[\operatorname{Im}\left(f_{l}\right)+\mathcal{O}(\gamma)\right]}{3\left|f_{l}\right|^{2} \Lambda_{l}^{\prime}\left(z_{0}\right) \gamma^{2}}+\text { nonsingular terms; }
$$

finally, the $\Gamma_{3,0}$ factor is generically nonzero and can be evaluated from (75) as

$$
\Gamma_{3,0}=-2\left(\pi K f_{l}^{*}\right)^{2} \frac{\Lambda_{l}^{\prime \prime}\left(z_{0}\right)}{\Lambda_{3 l}\left(z_{0}\right)} .
$$

Hence $p_{2} \sim \gamma^{-2}$ for the special case $f_{2 l}=0$ when $p_{1}$ is nonsingular. However, if $f_{2 l}$ and $f_{3 l}$ both vanish, then the divergent terms in (102) disappear and both $p_{1}$ and $p_{2}$ are nonsingular. 
These conclusions are generalized below. In Proposition 7.1 we show that when $f_{2 l}=0$ but $f_{3 l} \neq 0$, then $p_{j} \sim \gamma^{-2 j+2}$ describes the strongest possible singularity of $p_{j}$. In Proposition 7.2 we find that the coefficients $p_{j}$ are nonsingular at every order if $f_{n l}=0$ for all $n>1$.

The implications of the $p_{2}$ divergence for the scaling of the amplitude, $\gamma^{\beta} r(\gamma t)$, can be seen by rewriting the radial equation (91) to allow for both the third and fifth order terms:

$$
\frac{d r}{d \tau}=\gamma r+\operatorname{Re}\left(p_{1}\right) \gamma^{2 \beta-1} r^{3}+\operatorname{Re}\left(p_{2}\right) \gamma^{4 \beta-1} r^{3}
$$

When $p_{1}$ is nonsingular and $p_{2} \sim \gamma^{-2}$, then we must take $\beta=3 / 4$ to obtain an expansion that is finite through fifth order terms. In this case however the general singularity result $p_{j} \sim \gamma^{-2 j+2}$ in Proposition 7.1 does not exclude higher order terms that would require a still larger value of $\beta$; this point is discussed further in Section 7.2 below.

\section{Singularity structure of the expansion to all orders}

Our goal is a systematic understanding of the singularities in $p_{j}$ to all orders in the amplitude expansion. These singularities are smoothed by the noise (cf. the singularity in (85)), and in the discussion below we always set $D=0$. In this analysis, it is useful to introduce an index which keeps track of the worst case singularity for each coefficient.

\subsection{Definition of the index}

The pinching singularities at every order occur in integrands of a standard form. Let

$$
D_{n}(\alpha, \omega) \equiv \frac{1}{\left(\omega-\alpha_{1}\right)\left(\omega-\alpha_{2}\right) \cdots\left(\omega-\alpha_{n}\right)}
$$

for $n>0$ where $\alpha \equiv\left(\alpha_{1}, \ldots, \alpha_{n}\right)$ and define $D_{0}(\alpha, \omega) \equiv 1$. Evaluating $p_{j}$ for $j \geq 1$ involves integrals of the form $\int d \omega \phi(\omega) \mathcal{G}(\omega)$ where

$$
\mathcal{G}(\omega)=D_{m}(\beta, \omega)^{*} D_{n}(\alpha, \omega)
$$


with $m+n \geq 1$. Here $\phi(\omega)$ denotes a smooth function assumed to be well behaved as $\gamma \rightarrow 0$ and to vanish for $|\omega| \rightarrow \infty$. For example, $\phi(\omega)=g(\omega)$ is often the smooth function of interest. The poles in (106) are given by

$$
\begin{array}{ll}
\alpha_{j}=z_{0}-K f_{0}+i \gamma \delta_{j} & j=1, \ldots, n \\
\beta_{j}^{*}=z_{0}^{*}-K f_{0}-i \gamma \zeta_{j} & j=1, \ldots, m ;
\end{array}
$$

hence they lie along the vertical line $\omega=\operatorname{Re}\left(z_{0}\right)-K f_{0}$ at locations determined by the numbers $\delta_{j} \geq 0$ and $\zeta_{j} \geq 0$ which are assumed to be independent of $\gamma=l \operatorname{Im}\left(z_{0}\right)$.

The index of $\mathcal{G}(\omega)$ in (106) is defined to be

$$
\text { Ind }[\mathcal{G}] \equiv m+n-1
$$

We extend this definition to assign an index if $\mathcal{G}(\omega)$ is multiplied by a smooth function $\phi(\omega)$ as described above

$$
\text { Ind }[\phi(\omega) \mathcal{G}(\omega)] \equiv \text { Ind }[\mathcal{G}(\omega)] \text {. }
$$

Since we assume $m+n \geq 1$ in (106), Ind $[\phi \mathcal{G}] \geq 0$. If $m n \neq 0$, then as $\gamma \rightarrow 0^{+}$, the integral $\int d \omega \phi(\omega) \mathcal{G}(\omega)$ may diverge due to a pinching singularity at $\omega=\operatorname{Re}\left(z_{0}\right)-K f_{0}$. The smooth functions $\phi(\omega)$ are always assumed to be sufficiently well behaved at large $|\omega|$ that the only possible divergence is due to the pinching singularity. The index of $\phi \mathcal{G}$ is simply related to the maximum possible strength of this divergence.

Proposition 6.1 For $\mathcal{G}(\omega)$ in (106) with $m+n \geq 1$ and $\phi(\omega)$ a smooth function as described above, the integral of $\phi \mathcal{G}$ satisfies

$$
\lim _{\gamma \rightarrow 0^{+}}\left|\gamma^{J} \int_{-\infty}^{\infty} d \omega \phi(\omega) \mathcal{G}(\omega)\right|<\infty
$$

with $J=\operatorname{Ind}[\phi \mathcal{G}]$. If $J$ is replaced by $J-1$, then the limit (111) diverges in general unless $m n=0$ in which case the limit is zero for any $J>0$. 


\section{Proof.}

See Appendix A.

Our application of Prop. 6.1 to the recursion relations for $I_{m, j}$ requires a further generalization of the index to allow for sums of functions with well-defined indices and products of $\phi \mathcal{G}$ with singular functions of $\gamma$. In each case, the generalized index is defined so that Eq. (111) remains true, i.e. the index for the composite function gives the maximal possible divergence of its integral.

First, let $G_{1}(\omega)$ and $G_{2}(\omega)$ denote functions with well-defined indices, Ind $\left[G_{1}\right] \geq \operatorname{Ind}\left[G_{2}\right]$. The index of their sum is defined as the largest of the two individual indices:

$$
\text { Ind }\left[G_{1}+G_{2}\right] \equiv \text { Ind }\left[G_{1}\right]
$$

Clearly, Ind $\left[G_{1}\right]$ gives the maximal possible divergence of $\int d \omega\left(G_{1}+G_{2}\right)$. Secondly, let $q(\gamma)$ denote a function of $\gamma$ with the asymptotic behavior

$$
\lim _{\gamma \rightarrow 0^{+}} q(\gamma) \sim \gamma^{-\nu}
$$

then the index of $q(\gamma) G_{1}(\omega)$ is defined by

$$
\text { Ind }\left[q G_{1}\right] \equiv \text { Ind }\left[G_{1}\right]+\nu \text {. }
$$

This completes the definition of the index.

By applying (112) and (114) the indices of $I_{m, j}$ and $h_{m, j}$ are determined from the recursion relations; some simple examples are provided in section 6.2. We stress again that when applied to a composite function $G(\omega)$ the estimate in (111) does not necessarily determine the true singularity, but only an upper bound on the possible divergence of the integral. In many examples considered below this upper bound is actually realized, but not always. 
There are two immediate implications of the index definition worth noting. First, complex conjugation doesn't change the index

$$
\text { Ind }[G]=\operatorname{Ind}\left[G^{*}\right] \text {. }
$$

Secondly, if $G(\omega)$ has a well defined index, then dividing $G$ by $(\omega-\alpha)$ or $\left(\omega-\beta^{*}\right)$ simply increases the index of $G$ by one:

$$
\text { Ind }[G /(\omega-\alpha)]=\text { Ind }\left[G /\left(\omega-\beta^{*}\right)\right]=\text { Ind }[G]+1 \text {. }
$$

Here $\alpha, \beta$ introduce additional poles as defined in (107) - (108) and thereby increase the index of every term in $G$ by one; this implies (116).

The second observation (116) provides a useful relation between the divergence of $\Gamma_{m, j}$ and the divergence of $\left\langle\tilde{\psi}, h_{m, j}>\right.$. Suppose that $\gamma^{-\nu}, \nu>0$, denotes the actual divergence of $\Gamma_{m, j}$. Then, from (75) this must be due to certain terms in $I_{m, j}$; in addition when $m=1$ then $\Lambda_{l}\left(\nu_{1, j}\right)$ is of order $\gamma$ for small $\gamma$, which increases the divergence by one. From (76), $<\tilde{\psi}, h_{m, j}>$ is given by

$$
\begin{aligned}
<\tilde{\psi}, h_{m, j}>= & \frac{-i}{m l \Lambda_{l}^{\prime}\left(z_{0}\right)} \int_{-\infty}^{\infty} \frac{d \omega g(\omega) I_{m, j}(\omega)}{\left(\omega+K f_{0}-z_{0}\right)\left(\omega+K f_{0}-\nu_{m, j}\right)} \\
& +\frac{K f_{m l}^{*} \Gamma_{m, j}}{\Lambda_{l}^{\prime}\left(z_{0}\right)} \int_{-\infty}^{\infty} \frac{d \omega g(\omega)}{\left(\omega+K f_{0}-z_{0}\right)\left(\omega+K f_{0}-\nu_{m, j}\right)},
\end{aligned}
$$

and we conclude that its divergence cannot be worse than $\gamma^{-(\nu+1-\delta m, 1)}$. The second integral in (117) has no pinching singularity and the singularity of the second term is determined by $\Gamma_{m, j} \sim \gamma^{-\nu}$. The first term in (117) involves an integral whose integrand differs from the integrand in (76) only by the extra factor $\left(\omega+K f_{0}-z_{0}\right)$ in the denominator. From (116) this factor simply increases the index of every term in $I_{m, j}(\omega) /\left(\omega+K f_{0}-\nu_{m, j}\right)$. In particular, the terms that determined the singularity of $\Gamma_{m, j} \sim \gamma^{-\nu}$ in the first place have their index 
increased by 1 . Thus we can infer the divergence of the first term in (117) by adding one to $\nu$ and subtracting off the effect of $\Lambda_{m l}\left(\nu_{m, j}\right)$ if $m=1$; this proves that the singularity of $<\tilde{\psi}, h_{m, j}>$ is no worse than $\gamma^{-(\nu+1-\delta m, 1)}$. Note that if $\Gamma_{m, j}$ is not singular $(\nu=0)$ then the same reasoning shows that $<\tilde{\psi}, h_{m, j}>$ will also be nonsingular. In summary, we have proven

Proposition 6.2 If the asymptotic behavior of $\Gamma_{m, j}$ satisfies

$$
\lim _{\gamma \rightarrow 0^{+}} \gamma^{\nu}\left|\Gamma_{m, j}\right|<\infty
$$

for an integer $\nu>0$, then

$$
\lim _{\gamma \rightarrow 0^{+}} \gamma^{\nu^{\prime}}\left|<\tilde{\psi}, h_{m, j}>\right|<\infty
$$

with

$$
\nu^{\prime}=\left\{\begin{array}{cc}
\nu & m=1 \\
\nu+1 & m>1
\end{array}\right.
$$

If $\nu=0$ in (118), then

$$
\lim _{\gamma \rightarrow 0^{+}}\left|<\tilde{\psi}, h_{m, j}>\right|<\infty .
$$

This result is useful because $\nu^{\prime}$ in (120) often turns out to be less than the index of the integrand of $<\tilde{\psi}, h_{m, j}>$ and thus the Proposition can provide sharper estimates of the actual divergences.

It is very important to recognize that the conclusions in (119) and (121) do not in general apply to $\left\langle\tilde{\psi}, h_{m, j}^{*}>\right.$. Even though $h_{m, j}$ and $h_{m, j}^{*}$ have the same index, the most singular terms in $\left\langle\tilde{\psi}, h_{m, j}>\right.$ are not necessarily the most singular terms in $<\tilde{\psi}, h_{m, j}^{*}>$. For $\left\langle\tilde{\psi}, h_{m, j}^{*}>\right.$, if we wish to avoid explicitly evaluating the integral, our only means of estimating the divergence is by calculating the index of the integrand. 


\subsection{Calculation of the index}

These definitions allow the index of $I_{m, j}$ and $h_{m, j}$ to be calculated recursively to all orders. We illustrate this index calculation for the first few levels of Table I; our most general results are stated in Theorem 6.1 and Proposition 7.1.

At the top of Table I we have the eigenfunctions defined by linear theory, these have a single pole in the upper half plane so

$$
\text { Ind }[\psi(\omega)]=\text { Ind }[\tilde{\psi}(\omega)]=0 \text {. }
$$

Next we consider $I_{2,0}$ and $h_{2,0}$; from (79) and (83) we calculate

$$
\text { Ind }\left[I_{2,0}(\omega)\right]=0 \quad \text { Ind }\left[h_{2,0}(\omega)\right]=1
$$

The index of $I_{2,0}$ follows from (122), while the expression for $h_{2,0}$ has two terms; the first has index 1 and the second has index 0 , hence the index of $h_{2,0}$ is 1 .

Since $h_{2,0}$ only has poles in the upper half plane, the integrals $\Gamma_{2,0}$ and $<\tilde{\psi}, h_{2,0}>$ have no pinching singularities and remain finite as $\gamma \rightarrow 0^{+}$:

$$
\Gamma_{2,0} \sim \gamma^{0} \quad<\tilde{\psi}, h_{2,0}>\sim \gamma^{0}
$$

Note that the integrand in (124) has index equal to 2, but there is no divergence, this

illustrates the second part of Proposition 6.2. By contrast the integrand of $<\tilde{\psi}, \psi^{*}>$ has a smaller index than $<\tilde{\psi}, h_{2,0}>$ but yields a singular integral (85) as $\gamma \rightarrow 0^{+}$:

$$
<\tilde{\psi}, \psi^{*}>\sim \gamma^{-1}
$$

The fifth order coefficient $p_{2}$ provides an instructive exercise in applying the index to estimate the singularity of a more complicated expression. The general form of the coefficient 
(not assuming $f_{2 l}=0$ ) is

$$
\begin{aligned}
p_{2}=-(2 \pi i K l)\{ & f_{l}\left[<\tilde{\psi}, h_{2,1}>+\Gamma_{1,0}^{*}<\tilde{\psi}, h_{2,0}>\right]+f_{2 l} \Gamma_{2,0}^{*}<\tilde{\psi}, h_{3,0}> \\
& \left.+f_{2 l}^{*}\left[\Gamma_{2,1}<\tilde{\psi}, \psi^{*}>+\Gamma_{2,0}<\tilde{\psi}, h_{1,0}^{*}>\right]+f_{3 l}^{*} \Gamma_{3,0}<\tilde{\psi}, h_{2,0}^{*}>\right\}
\end{aligned}
$$

where

$$
\begin{aligned}
I_{3,0} & =2 \pi i K(3 l)\left[f_{l}^{*} h_{2,0}+f_{2 l}^{*} \psi \Gamma_{2,0}\right] \\
I_{1,0} & =(2 \pi i K l) \mathrm{P}_{\perp}\left\{f_{l} h_{2,0}+f_{2 l}^{*} \psi^{*} \Gamma_{2,0}\right\} \\
I_{2,1} & =2 p_{1} h_{2,0}+2 \pi i K(2 l)\left[f_{l} h_{3,0}+f_{l}^{*}\left(h_{1,0}+\Gamma_{1,0} \psi\right)+f_{3 l}^{*} \Gamma_{3,0} \psi^{*}\right] .
\end{aligned}
$$

We can immediately estimate the integral $<\tilde{\psi}, h_{2,0}^{*}>$ from the fact that the integrand has index 2

$$
<\tilde{\psi}, h_{2,0}^{*}>\sim \gamma^{-2}
$$

The two terms in $I_{3,0}$ have indices of 1 and 0 , respectively, and the poles of $I_{3,0}$ are all in the upper half plane. Writing out the expression in (128)

$$
\mathrm{P}_{\perp}\left\{f_{l} h_{2,0}+f_{2 l}^{*} \psi^{*} \Gamma_{2,0}\right\}=f_{l} h_{2,0}+f_{2 l}^{*} \psi^{*} \Gamma_{2,0}-\psi<\tilde{\psi},\left[f_{l} h_{2,0}+f_{2 l}^{*} \psi^{*} \Gamma_{2,0}\right]>
$$

we find terms of index 1,0 , and 1 , respectively. The third term is already singular due to $<\tilde{\psi}, \psi^{*}>$ and the second term has a pole in the lower half plane. These observations imply

$$
\text { Ind }\left[I_{3,0}(\omega)\right]=1 \quad \text { Ind }\left[I_{1,0}(\omega)\right] \leq 1
$$

and from (75)

$$
\Gamma_{1,0} \sim \gamma^{-2} \quad \Gamma_{3,0} \sim \gamma^{0}
$$

The inequality in (132) reflects the fact that there are two terms of index 1 and we have not ruled out the possibility of a cancellation. The indices of $h_{3,0}$ and $h_{1,0}$ are now easily evaluated from $(76)$

$$
\text { Ind }\left[h_{3,0}(\omega)\right]=2 \quad \text { Ind }\left[h_{1,0}(\omega)\right] \leq 2
$$


and the corresponding integrals are estimated following (119)

$$
<\tilde{\psi}, h_{3,0}>\sim \gamma^{0} \quad<\tilde{\psi}, h_{1,0}>\sim \gamma^{-2}
$$

For $p_{2}$ we also need to estimate $<\tilde{\psi}, h_{1,0}^{*}>$ and this is done by noting from (134) that the integrand has an index of (at most) 3 so

$$
<\tilde{\psi}, h_{1,0}^{*}>\sim \gamma^{-3}
$$

The five terms in $I_{2,1}$ all have index 2 except $\Gamma_{3,0} \psi^{*}$ which has index 0 , hence

$$
\text { Ind }\left[I_{2,1}(\omega)\right] \leq 2
$$

The terms involving $h_{1,0}$ and $\psi^{*}$ have poles in the lower half plane and therefore produce pinching singularities in $\Gamma_{2,1}$. By comparing (75) for $\Gamma_{2,1}$ to (125) and (135) we can estimate a maximum singularity of

$$
\Gamma_{2,1} \sim \gamma^{-2}
$$

which in turn implies Ind $\left[h_{2,1}(\omega)\right] \leq 3$, and

$$
<\tilde{\psi}, h_{2,1}>\sim \gamma^{-3}
$$

from (76) and (119), respectively.

The result in (139) gives the divergence of the first term in $p_{2}$, the asymptotic behavior of the remaining five terms can be evaluated from the foregoing estimates:

$$
\begin{aligned}
\Gamma_{1,0}^{*}<\tilde{\psi}, h_{2,0}> & \sim \gamma^{-2} \\
\Gamma_{2,0}^{*}<\tilde{\psi}, h_{3,0}> & \sim \gamma^{0} \\
\Gamma_{2,1}<\tilde{\psi}, \psi^{*}> & \sim \gamma^{-3} \\
\Gamma_{2,0}<\tilde{\psi}, h_{1,0}^{*}> & \sim \gamma^{-3} \\
\Gamma_{3,0}<\tilde{\psi}, h_{2,0}^{*}> & \sim \gamma^{-2} .
\end{aligned}
$$


Hence barring some accidental cancellation among the most divergent terms we expect $p_{2} \sim$ $\gamma^{-3}$ for the general case when $f_{2 l} \neq 0$; more precisely we have proved

$$
\lim _{\gamma \rightarrow 0^{+}} \gamma^{3}\left|p_{2}\right|<\infty
$$

\subsection{The main result}

Our main result on the singularity structure of the amplitude expansions can now be proved. In the preceeding sections, we have calculated the indices of $I_{m, j}$ and $h_{m, j}$ through the first two levels of Table I, and determined the singular behavior of the cubic and fifth order coefficients. This information is now inferred for the entire theory to all orders.

Theorem 6.1 For $j \geq 1$, the singularities of the coefficients in the amplitude expansion satisfy

$$
\lim _{\gamma \rightarrow 0^{+}} \gamma^{2 j-1}\left|p_{j}\right|<\infty .
$$

For $m \geq 1$ and $j \geq 0$, the indices of $I_{m, j}$ and $h_{m, j}$ obey

$$
\begin{aligned}
& \text { Ind }\left[I_{m, j}\right] \leq J_{m, j}+2 \delta_{m, 1} . \\
& \text { Ind }\left[h_{m, j}\right] \leq J_{m, j}+1+2 \delta_{m, 1}
\end{aligned}
$$

where $J_{m, j} \equiv m+2 j-2$, and the integrals in (70) satisfy

$$
\begin{aligned}
\lim _{\gamma \rightarrow 0^{+}} \gamma^{J_{m, j}+3 \delta_{m, 1}}\left|\Gamma_{m, j}\right| & <\infty \\
\lim _{\gamma \rightarrow 0^{+}} \gamma^{J_{m, j}+1+2 \delta_{m, 1}}\left|<\tilde{\psi}, h_{m, j}>\right| & <\infty .
\end{aligned}
$$

We are only able to prove the results in (147) - (148) as upper bounds on the index, but we expect them to hold as equalities in most cases. Note that the bounds in (149) - (150) give weaker divergences than one would estimate simply from the index of the integrand as

calculated using (147) - (148). For example, from (148) the integrand of $<\tilde{\psi}, h_{m, j}>$ has 
index $J_{m, j}+2+2 \delta_{m, 1}$, but the bound in (150) assures us that the divergence of this integral will always be at least one power of $\gamma$ less. There is no contradiction since the index only gives a strict upper limit on divergence exhibited by a given integral.

\section{Proof.}

1. The relations (146) - (150) have been checked explicitly for $I_{2,0}, h_{2,0}, \Gamma_{2,0}$, and $p_{1}$ in the analysis of section 5.1. In addition, in section 6.2 they have been verified for $I_{1,0}, h_{1,0}, \Gamma_{1,0}, I_{3,0}, h_{3,0}, \Gamma_{3,0}, I_{2,1}, h_{2,1}, \Gamma_{2,1}$, and $p_{2}$. This proves the theorem for the first two levels of Table I.

2. We extend (146) - (150) to all $p_{j}$, and $\left\{h_{m, j}, I_{m, j}\right\}$ by induction using the recursion relations. Assume that (146) - (150) are true down to some arbitrary level of Table I, and consider what the recursion relations imply for the coefficients evaluated at the next level. Let $h_{m^{\prime}, j^{\prime}}$ and $I_{m^{\prime}, j^{\prime}}$ denote coefficients at the next level that can be evaluated from lower order quantities which satisfy (146) - (150). We first consider the various recursion relations for $h_{m^{\prime}, j^{\prime}}$ and $I_{m^{\prime}, j^{\prime}}$, and prove that if (146) - (150) hold on the right hand side of these relations, then (147) - (150) will also hold for the coefficients obtained on the left. We organize this part of the proof by noting the importance of establishing two relations for $I_{m^{\prime}, j^{\prime}}$ :

(a) the index identity (147)

$$
\text { Ind }\left[I_{m^{\prime}, j^{\prime}}\right] \leq J_{m^{\prime}, j^{\prime}}+2 \delta_{m^{\prime}, 1}
$$

(b) the estimate

$$
\lim _{\gamma \rightarrow 0^{+}} \gamma^{J_{m^{\prime}, j^{\prime}}+2 \delta_{m^{\prime}, 1}}\left|\int_{-\infty}^{\infty} d \omega \frac{g(\omega) I_{m^{\prime}, j^{\prime}}(\omega)}{(\omega-\alpha)}\right|<\infty
$$


where $\alpha$ is any pole of the form described in (107) (in the upper half plane). Note that (151) implies that the integrand in (152) has an index of $J_{m^{\prime}, j^{\prime}}+2 \delta_{m^{\prime}, 1}+1$; thus (152) gives a divergence which is less by one power of $(1 / \gamma)$ than would be "naively" predicted using the index of $I_{m^{\prime}, j^{\prime}}$ in (151).

If we can prove (151) and (152) for $I_{m^{\prime}, j^{\prime}}$, then the remaining properties (148) - (150) are easily obtained as follows. First, (149) follows from (152) and the identity (75). Next (148) follows by applying (151) and (149) to the identity (76). Finally (150) follows from Proposition 6.2 and (149). Thus the crux of the matter is to verify (151) and (152); this is done using the recursion relations for $I_{m^{\prime}, j^{\prime}}$, i.e. using (77) - (81).

3. Verification of (151).

The index of each term on the right hand side of the recursion relations for $I_{m^{\prime}, j^{\prime}}(\omega)$ can be evaluated by applying (146) - (150); this exercise shows that all of these terms have an index less than or equal to $J_{m^{\prime}, j^{\prime}}+2 \delta_{m^{\prime}, 1}$. This establishes (151) for $I_{m^{\prime}, j^{\prime}}$. A few examples from (77) illustrate these index calculations.

(a) The terms in $I_{1, j}$ that depend on $p_{j}$ have the form $\left[(2+k) p_{j-k}+(1+\right.$ $\left.k) p_{j-k}^{*}\right] h_{1, k}$; from (148) we have Ind $\left[h_{1, k}\right] \leq 2 k+2$ and from (146) the singularity of $p_{j-k}$ is determined, hence

$$
\text { Ind }\left[p_{j-k} h_{1, k}\right] \leq 2(j-k)-1+(2 k+2)=2 j+1
$$

which is consistent with (151) for $\left(m^{\prime}, j^{\prime}\right)=(1, j)$. 
(b) Next consider the terms

$$
\sum_{m=3}^{j+2} f_{m l}^{*} \sum_{n=0}^{j-m+2} \mathrm{P}_{\perp}\left[h_{m-1, n}^{*} \Gamma_{m, j-m-n+2}\right]
$$

which expand to

$$
\mathrm{P}_{\perp}\left[h_{m-1, n}^{*} \Gamma_{m, j-m-n+2}\right]=\Gamma_{m, j-m-n+2}\left[h_{m-1, n}^{*}-<\tilde{\psi}, h_{m-1, n}^{*}>\psi\right] .
$$

The index of each term can be evaluated

$$
\begin{aligned}
\text { Ind }\left[h_{m-1, n}^{*} \Gamma_{m, j-m-n+2}\right] & \leq 2 j \\
\text { Ind }\left[<\tilde{\psi}, h_{m-1, n}^{*}>\Gamma_{m, j-m-n+2} \psi\right] & \leq 2 j+1 ;
\end{aligned}
$$

thus Ind $\left[\mathrm{P}_{\perp}\left[h_{m-1, n}^{*} \Gamma_{m, j-m-n+2}\right]\right] \leq 2 j+1$ which is consistent with (151) for $\left(m^{\prime}, j^{\prime}\right)=(1, j)$. In this example, both indicies are obtained from (148) and (149). The divergence of $\Gamma_{m, j-m-n+2} \sim \gamma^{-(2 j-m-2 n+2)}$ is estimated from (149), and (148) gives Ind $\left[h_{m-1, n}^{*}\right] \leq m+2 n-2$. Hence the integrand of $<\tilde{\psi}, h_{m-1, n}^{*}>$ has an index no greater than $m+2 n-1$, and we conclude that the worst possible divergence is $<\tilde{\psi}, h_{m-1, n}^{*}>\sim \gamma^{-(m+2 n-1)}$. These estimates immediately imply (156) - (157).

(c) A more subtle example is the term $\mathrm{P}_{\perp} h_{2, j}$ in (77); from (61) we have that the index of $\mathrm{P}_{\perp} h_{2, j}=h_{2, j}-\psi<\tilde{\psi}, h_{2, j}>$ is equal to the largest index obtained from $h_{2, j}$ and $<\tilde{\psi}, h_{2, j}>\psi(\omega)$. These terms have indices

$$
\text { Ind }\left[h_{2, j}\right] \leq 2 j+1 \quad \text { Ind }\left[<\tilde{\psi}, h_{2, j}>\psi(\omega)\right] \leq 2 j+1,
$$

so Ind $\left[\mathrm{P}_{\perp} h_{2, j}\right] \leq 2 j+1$ which is consistent with (151) for $\left(m^{\prime}, j^{\prime}\right)=$ $(1, j)$. The first index in (158) comes from (148) and the second follows 
from (150) and (123). This example is delicate because (158) requires that we use (150) to estimate $<\tilde{\psi}, h_{2, j}>\sim \gamma^{-(2 j+1)}$ rather than (148) as in the previous calculation (157).

Proceeding in this way the index of every term in the recursion relations (87) - (81) can be calculated and (151) verified.

4. Verification of (152).

We prove (152) by integrating the recursion relations for $I_{m^{\prime}, j^{\prime}}(\omega)$ and examining the resulting recursion relations for

$$
\int_{-\infty}^{\infty} d \omega \frac{g(\omega) I_{m^{\prime}, j^{\prime}}(\omega)}{(\omega-\alpha)}
$$

The right hand side of the recursion relations (77) - (81) involve products of $\psi, \psi^{*}, h_{m, j}$, or $h_{m, j}^{*}$ with integrals whose singularities can be estimated by applying (146) - (150) as illustrated above. Now when we divide by $(\omega-\alpha)$ and integrate as in (159) we get additional integrals of the following types

$$
\begin{array}{cc}
\int_{-\infty}^{\infty} d \omega \frac{g(\omega) \psi(\omega)}{(\omega-\alpha)} & \int_{-\infty}^{\infty} d \omega \frac{g(\omega) \psi^{*}(\omega)}{(\omega-\alpha)} \\
\int_{-\infty}^{\infty} d \omega \frac{g(\omega) h_{m, j}^{*}(\omega)}{(\omega-\alpha)} & \int_{-\infty}^{\infty} d \omega \frac{g(\omega) h_{m, j}(\omega)}{(\omega-\alpha)} .
\end{array}
$$

The first integral in (160) has no pinching singularity and the second has index 1 and diverges as $\gamma^{-1}$; the singularity of the integrals in (161) can be estimated from (148) and (150), respectively:

$$
\begin{aligned}
& \lim _{\gamma \rightarrow 0^{+}} \gamma^{J_{m, j}+2+2 \delta_{m, 1}}\left|\int_{-\infty}^{\infty} d \omega \frac{g(\omega) h_{m, j}^{*}(\omega)}{(\omega-\alpha)}\right|<\infty \\
& \lim _{\gamma \rightarrow 0^{+}} \gamma^{J_{m, j}+1+2 \delta_{m, 1}}\left|\int_{-\infty}^{\infty} d \omega \frac{g(\omega) h_{m, j}(\omega)}{(\omega-\alpha)}\right|<\infty .
\end{aligned}
$$


With these estimates the terms contributing to (159) can be shown to diverge at worst like

$$
\left(\frac{1}{\gamma}\right)^{J_{m^{\prime}, j^{\prime}}+2 \delta_{m^{\prime}, 1}}
$$

This establishes (152) for $I_{m^{\prime}, j^{\prime}}$, and completes the induction argument that (147) - (150) are propagated by the recursion relations.

5. Now we finish the proof of the theorem by verifying that the estimates for the integrals in (149) and (150) imply the estimate of $p_{j}$ in (146); this is a straightforward application of (149) and (150) to the right hand side of the recursion relations (70). A few examples from (70) suffice to illustrate how this is done.

(a) Consider the term $<\tilde{\psi}, h_{2, j-1}>$; from (150) the singularity of this integral is at most

$$
\left|<\tilde{\psi}, h_{2, j-1}>\right| \sim\left(\frac{1}{\gamma}\right)^{J_{2, j-1}+1}=\left(\frac{1}{\gamma}\right)^{2 j-1}
$$

which is consistent with (146). This example is another case where the divergence of the integral is best characterized with (150) rather than inferred from the index of the integrand which is $2 j$.

(b) Other terms involve products of integrals, for example, $\Gamma_{2, j-1}<\tilde{\psi}, \psi^{*}>$. Using (149) and (125) we determine that the singularity of this product is at most

$$
\left|\Gamma_{2, j-1}<\tilde{\psi}, \psi^{*}>\right| \sim\left(\frac{1}{\gamma}\right)^{J_{2, j-1}+1}
$$

which is the same as in (165).

(c) The terms

$$
\sum_{m=3}^{j+1} f_{m l}^{*} \sum_{n=0}^{j-m+1} \Gamma_{m, n}<\tilde{\psi}, h_{m-1, j-n-m+1}^{*}>\text {; }
$$


are a final example. Applying (148) and (149) shows a maximum singularity of

$$
\left|\Gamma_{m, n}<\tilde{\psi}, h_{m-1, j-n-m+1}^{*}>\right| \sim\left(\frac{1}{\gamma}\right)^{J_{m, n}+J_{m-1, j-n-m+1}+2}=\left(\frac{1}{\gamma}\right)^{2 j-1}
$$

which is also consistent with (146).

In this manner every term in the recursion relation for $p_{j}$ is shown to satisfy the general estimate in (146).

\subsection{Implications of Theorem 6.1}

Our discussion in section 5.1 of the significance of the coefficient singularities did not consider terms in the amplitude equation beyond third order. If all such terms are included then the amplitude equation in polar variables (90) becomes

$$
\begin{aligned}
& \dot{A}=\gamma A+\sum_{j=1}^{\infty} \operatorname{Re}\left(p_{j}\right) A^{2 j+1} \\
& \dot{\psi}=\Omega-\sum_{j=1}^{\infty} \operatorname{Im}\left(p_{j}\right) A^{2 j}
\end{aligned}
$$

With the asymptotic behavior of the coefficients is given by Theorem 6.1

$$
p_{j}=\frac{\left[a_{j}+\mathcal{O}(\gamma)\right]}{\gamma^{2 j-1}}
$$

we re-express the dynamics in terms of the rescaled amplitude (7) $A(t)=\gamma^{\beta} r\left(\gamma^{\delta} t\right)$

$$
\begin{aligned}
\frac{d r}{d \tau} & =\gamma^{1-\delta} r+\sum_{j=1}^{\infty} \gamma^{2 j(\beta-1)-\delta+1} \operatorname{Re}\left(a_{j}\right)[1+\mathcal{O}(\gamma)] r^{2 j+1} \\
\frac{d \psi}{d t} & =\Omega-\sum_{j=1}^{\infty} \gamma^{2 j(\beta-1)+1} \operatorname{Im}\left(a_{j}\right)[1+\mathcal{O}(\gamma)] r^{2 j}
\end{aligned}
$$


Now we see that the singularities of $p_{j}$ are such that the choices $\delta=1$ and $\beta=1$ remove them to all orders, leaving an asymptotically finite system

$$
\begin{aligned}
& \frac{d r}{d \tau}=r+\sum_{j=1}^{\infty} \operatorname{Re}\left(a_{j}\right)[1+\mathcal{O}(\gamma)] r^{2 j+1} \\
& \frac{d \psi}{d t}=\Omega-\gamma \sum_{j=1}^{\infty} \operatorname{Im}\left(a_{j}\right)[1+\mathcal{O}(\gamma)] r^{2 j}
\end{aligned}
$$

In (174) there is a further remarkable consequence of the singularities, namely that the terms at higher order in $r$ no longer appear to be negligible even for small $\gamma$. This conclusion is provisional in the sense that we have not proven $a_{j} \neq 0$ at high order. Nevertheless, our analysis of $p_{1}$ and $p_{2}$ shows that in general $a_{1}$ and $a_{2}$ are non-zero, and inspection of the recursion relations shows no evidence that the series will truncate. This feature does not affect the phase evolution (175) quite so dramatically. If we assume a time-independent solution $r=R_{r w}$ of (174) then the nonlinear frequency of this state

$$
\Omega_{r w}=\Omega-\gamma \sum_{j=1}^{\infty} \operatorname{Im}\left(a_{j}\right)[1+\mathcal{O}(\gamma)] R_{r w}^{2 j}
$$

is very close to the linear frequency $\Omega$ for small $\gamma$. Such solutions are called "rotating wave" states as they describe a solution (65)

$$
\begin{aligned}
\eta^{u}(\theta, \omega, t)= & {\left[\gamma^{\beta} R_{r w} e^{-i \Omega_{r w} t} \Psi(\theta, \omega)+\gamma^{3 \beta} R_{r w}^{3} e^{-i \Omega_{r w} t} h_{1}\left(\omega, \gamma^{2 \beta} R_{r w}^{2}\right) e^{i l \theta}\right.} \\
& \left.+\sum_{m=2}^{\infty} \gamma^{m \beta} R_{r w}^{m} e^{-i m \Omega_{r w} t} h_{m}\left(\omega, \gamma^{2 \beta} R_{r w}^{2}\right) e^{i m l \theta}\right]+ \text { c.c. }
\end{aligned}
$$

that appears time independent in a rotating frame $\theta^{\prime}=\theta-\Omega_{r w} t / l$.

The asymptotic behavior of $\Gamma_{m, j}$ is given by Theorem 6.1 as

$$
\Gamma_{m, j}=\frac{\left[b_{m, j}+\mathcal{O}(\gamma)\right]}{\gamma^{J_{m, j}+3 \delta_{m, 1}}}
$$

where the constants $b_{m, j}$ are independent of $\gamma$. The expansion of $\Gamma_{m}$ thus has the asymptotic form

$$
\Gamma_{m}(\sigma)=\sum_{j=0}^{\infty} \Gamma_{m, j} \sigma^{j}=\left(\frac{1}{\gamma}\right)^{m-2+3 \delta_{m, 1}} \sum_{j=0}^{\infty}\left[b_{m, j}+\mathcal{O}(\gamma)\right] r^{2 j}
$$


This motivates the definition of the nonsingular coefficient $\hat{\Gamma}_{m}$

$$
\Gamma_{m}(\sigma) \equiv\left(\frac{1}{\gamma}\right)^{m-2+3 \delta_{m, 1}} \hat{\Gamma}_{m}\left(r^{2}\right) ;
$$

$\hat{\Gamma}_{m}$ will play a role shortly in our discussion of Daido's order parameter.

\section{Special Cases: $f_{2 l}=0$}

The general analysis shows that if $f_{2 l} \neq 0$, then the resulting singularities require a rescaling of the amplitude by a factor of $\gamma$. In this section, we consider some of the possiblities that can occur for couplings with $f_{2 l}=0$. First, we note that the general recursion relations simplify somewhat when $f_{2 l}=0$; the terms proportional to $f_{2 l}$ are explicitly shown in (70) and (77) - (81), and we simply drop them.

\subsection{Absence of the cubic singularity: $f_{2 l}=0$ and $f_{3 l} \neq 0$}

When $f_{2 l}=0$ so that the cubic coefficient is non-singular, then if $f_{3 l} \neq 0$ the first singularity appears in the fifth order coefficient $p_{2} \sim \gamma^{-2}$. The general structure of the amplitude expansion for this situation is characterized by the following proposition.

Proposition 7.1 Suppose that $f_{2 l}=0$ and $f_{3 l} \neq 0$, then for $j \geq 1$, the singularities of the coefficients in the amplitude expansion satisfy

$$
\lim _{\gamma \rightarrow 0^{+}} \gamma^{2 j-2}\left|p_{j}\right|<\infty
$$

For $m \geq 1$ and $j \geq 0$, the indices of $I_{m, j}$ and $h_{m, j}$ obey

$$
\begin{aligned}
& \text { Ind }\left[I_{m, j}\right] \leq J_{m, j}+2 \delta_{m, 1} \\
& \text { Ind }\left[h_{m, j}\right] \leq J_{m, j}+1+2 \delta_{m, 1}
\end{aligned}
$$


where $J_{m, j} \equiv m+2 j-2$, and the integrals in (70) satisfy

$$
\begin{array}{r}
\lim _{\gamma \rightarrow 0^{+}} \gamma^{J_{m, j}+3 \delta_{m, 1}-\left(1-\delta_{m, 2} \delta_{j, 0}\right)}\left|\Gamma_{m, j}\right|<\infty \\
\lim _{\gamma \rightarrow 0^{+}} \gamma^{J_{m, j}+2 \delta_{m, 1}}\left|<\tilde{\psi}, h_{m, j}>\right|<\infty .
\end{array}
$$

Compared to the conclusions of Theorem 6.1 the bounds on the indices are the same so there is nothing to prove. The estimates of the singularities in (181) and (184) - (185) are softened by one factor of $\gamma$. Since $J_{2,0}=0$, there is no singularity in $\Gamma_{2,0}$ even in the general case and the odd correction term $\delta_{m, 2} \delta_{j, 0}$ in (184) adjusts the exponent to allow for this. In fact, $\Gamma_{m, 0}, m>1$ is nonsingular in general, although we have not used this.

\section{Proof.}

1. The results in (181) - (185) have been established by the calculations in sections 5.1 and 5.2; they are extended to all orders by induction. The argument is entirely analogous to the proof given for Theorem 6.1 and we indicate only the necessary modifications and omit the details.

2. The only adjustment required is that the second crucial identity (152) in the general proof is now replaced by the estimate

$$
\lim _{\gamma \rightarrow 0^{+}} \gamma^{J_{m^{\prime}, j^{\prime}}+2 \delta_{m^{\prime}, 1}-\left(1-\delta_{m^{\prime}, 2} \delta_{j^{\prime}, 0}\right)}\left|\int_{-\infty}^{\infty} d \omega \frac{g(\omega) I_{m^{\prime}, j^{\prime}}(\omega)}{(\omega-\alpha)}\right|<\infty
$$

where $\alpha$ is any pole of the form described in (107) (in the upper half plane). The first index identity (151) is still appropriate:

$$
\text { Ind }\left[I_{m^{\prime}, j^{\prime}}\right] \leq J_{m^{\prime}, j^{\prime}}+2 \delta_{m^{\prime}, 1}
$$

With this adjustment the repetition of the previous argument is straightforward. 


\subsection{Implications for scaling}

The consequences of this Theorem for the scaling of the amplitude are ambiguous. If we rewrite the radial equation from (172) with $\delta=1$

$$
\frac{d r}{d \tau}=r+\sum_{j=1}^{\infty} \gamma^{2 j \beta-1} \operatorname{Re}\left(p_{j}\right) r^{2 j+1},
$$

and then insert the estimated singularity $p_{j} \sim a_{j} \gamma^{-2 j+2}$ we find

$$
\frac{d r}{d \tau}=r+\sum_{j=1}^{\infty} \gamma^{2 j(\beta-1)+1} \operatorname{Re}\left(a_{j}\right) r^{2 j+1} .
$$

Now the requirement that all the nonlinear terms should be nonsingular, $2 j(\beta-1)+1 \geq 0$, results in a $j$-dependent estimate on the exponent: $\beta \geq 1-1 / 2 j$. We know the fifth order

singularity occurs so from $j=2$ we have $\beta=3 / 4$ as discussed previously in Section 5.2. However our analysis of the recursion relations is not sharp enough to determine if the leading singularities are actually present at every order. Consequently, we have a range of possible values of $\beta$

$$
\frac{3}{4} \leq \beta \leq 1 .
$$

This range can be reduced to $\beta=1$ if we require that the rescaling also yield a finite expansion for $\Gamma$ with coefficient singularities as estimated in (184). However it must be borne in mind that these singularities are upper bounds. Our firmest prediction remains of the range of values in (190) since the bound $\beta \geq 3 / 4$ is conclusively required by our fifth order calculations and the the bound $\beta \leq 1$ follows from our analysis of the general case.

\subsection{Single component couplings}

An additional case of interest is the circumstance which holds for the Kuramoto model $f(\phi)=\sin \phi$; a coupling that lacks all harmonics of the Fourier component $f_{l}$ driving the instability. In this exceptional case the amplitude expansion is entirely free of singularities. 
Proposition 7.2 If $f_{n l}=0$ for $n>1$, then the coefficients $p_{j}$ in (70) are all nonsingular:

$$
\lim _{\gamma \rightarrow 0^{+}}\left|p_{j}\right|<\infty
$$

for $j \geq 0$.

Proof.

The functions $I_{m, j}(\omega)$ and $h_{m, j}(\omega)$ have poles in the upper half plane only and consequently the integrals appearing in the coefficients $p_{j}$ are all free of pinching singularities in the limit $\gamma \rightarrow 0^{+}$. Also $\Gamma_{m, j}$ is nonsingular from (75) in light of the discussion following (96). Hence there are no singularities.

The absence of singularities results immediately in the conclusion $\beta=1 / 2$ for the scaling exponent.

\section{Evaluation of Daido's order function}

Daido's definition of the order function $H(\theta)$ is a direct generalization of the familiar order parameter of Kuramoto and is motivated by the mean field form of the phase dynamics (1). [13, 15, 34] He assumes there is a component of the population collectively entrained with frequency $\Omega_{e}$ and introduces the shifted phases $\psi_{j} \equiv \theta_{j}-\Omega_{e} t$, then phase equations (西) can be rewritten

$$
\dot{\psi}_{i}=\Delta_{i}-H\left(\psi_{i}, t\right)+\xi_{i}(t)
$$

where $\Delta_{i} \equiv \omega_{i}-\Omega_{e}$ and $H(\theta, t)$ is the mean field

$$
H(\theta, t) \equiv-\sum_{n=-\infty}^{\infty} f_{n} e^{-i n \theta}\left[\frac{1}{N} \sum_{j=1}^{N} e^{i n\left(\theta_{j}(t)-\Omega_{e} t\right)}\right] .
$$


The additive noise term $\xi_{i}(t)$ in (1) is omitted in the analysis, and for large $N$, Daido assumes $\Omega_{e}$ can be chosen so that the limit

$$
H(\theta) \equiv \lim _{t \rightarrow \infty} H(\theta, t)
$$

exists. The time-asymptotic mean field then defines the order function $H(\theta)$. A norm of $H$,

$$
\|H\|^{2}=\int_{0}^{2 \pi} d \theta H(\theta)^{2} / 2 \pi
$$

serves as an order parameter for models with multi-component couplings.

It is interesting to evaluate $H$ using our continuum description. The average over the population in (193) can be expressed in terms of $\rho(\theta, \omega, t)$ :

$$
\lim _{N \rightarrow \infty} \frac{1}{N} \sum_{j=1}^{N} e^{i n\left(\theta_{j}(t)-\Omega_{e} t\right)}=\int_{0}^{2 \pi} d \theta \int_{-\infty}^{\infty} d \omega g(\omega) \rho(\theta, \omega, t) e^{i n\left(\theta-\Omega_{e} t\right)},
$$

and this yields a general expression for the order function

$$
H(\theta) \equiv-\lim _{t \rightarrow \infty} \sum_{n=-\infty}^{\infty} f_{n} e^{-i n \theta} \int_{0}^{2 \pi} d \theta^{\prime} \int_{-\infty}^{\infty} d \omega^{\prime} g\left(\omega^{\prime}\right) \rho\left(\theta^{\prime}, \omega^{\prime}, t\right) e^{i n\left(\theta^{\prime}-\Omega_{e} t\right)}
$$

Let $H^{u}$ denote the order function obtained from solutions on the unstable manifold (65); a straightforward substitution from (65) then yields

$$
\begin{aligned}
H^{u}(\theta) / 2 \pi=- & \lim _{t \rightarrow \infty}\left\{f_{l} e^{-i l\left(\theta+\Omega_{e} t\right)}\left[\alpha(t)^{*}+\alpha(t)^{*} \sigma(t) \int_{-\infty}^{\infty} d \omega g(\omega) h_{1}(\omega, \sigma(t))^{*}\right]\right. \\
& \left.\left.+\sum_{m=2}^{\infty} f_{m l} e^{-i m l\left(\theta+\Omega_{e} t\right)}\left(\alpha(t)^{*}\right)^{m} \int_{-\infty}^{\infty} d \omega g(\omega) h_{m}(\omega, \sigma(t))^{*}\right)+c c\right\}
\end{aligned}
$$

note that we have set $f_{0}=0$ for simplicity.

Daido's assumption of time-independent behavior (194) is naturally satisfied when the system is described by a "rotating wave" solution of the form (cf. (177))

$$
\alpha_{r w}(t)=\gamma^{\beta} R_{r w} e^{-i \phi_{0}} e^{-i \Omega_{r w} t}
$$


with $R_{r w}$ and $\Omega_{r w}$ the time-independent amplitude and frequency, respectively. For these asymptotic states by choosing $\Omega_{e}=\Omega_{r w} / l$, the limit in (198) exists and the order function is

$$
\frac{H_{r w}^{u}}{2 \pi}=\left[f_{l}^{*} \gamma^{\beta} R_{r w} e^{-i \phi_{0}}\left(1+\gamma^{2 \beta} R_{r w}^{2} \Gamma_{1}\right) e^{i l \theta}+\sum_{m=2}^{\infty} f_{m l}^{*} \gamma^{m \beta} R_{r w}^{m} e^{-i m \phi_{0}} \Gamma_{m} e^{i m l \theta}\right]+\mathrm{cc}
$$

with norm given by

$$
\|H\|^{2}=(2 \pi)^{2} \gamma^{2 \beta} R_{r w}^{2}\left[\left|f_{l}\right|^{2}\left(1+\gamma^{2 \beta} R_{r w}^{2} \Gamma_{1}\right)^{2}+\sum_{m=2}^{\infty}\left|f_{m l}\right|^{2} \gamma^{(2 m-2) \beta} R_{r w}^{2 m-2} \Gamma_{m}^{2}\right] .
$$

Our results on the possible values of $\beta$ imply corresponding predictions for the scaling of $\|H\|$ near onset. There are several different cases depending on the coupling and whether or not noise is included; a summary appears in Table II.

1. Suppose that either $D>0$, or $D=0$ and we have a "single component" coupling: $f_{l} \neq 0$ and $f_{m l}=0$ for $m>1$. Then as $\gamma \rightarrow 0^{+}$, there are no singularities and (201) gives

$$
\|H\|=2 \pi \gamma^{\beta} R_{r w}\left|f_{l}\right|\left[1+\mathcal{O}\left(\gamma^{2 \beta}\right)\right] \sim\left(K-K_{c}\right)^{\beta}
$$

with $\beta=1 / 2$. In each of these circumstances, the prediction $\|H\| \sim\left(K-K_{c}\right)^{1 / 2}$ is in agreement with Daido's analysis of $\|H\|$.[15]

2. Suppose that $D=0$ and we have the general case with $f_{l} \neq 0$ and $f_{2 l} \neq 0$. The coefficients of the amplitude expansion have the generic singularities (146) and the coefficients $\Gamma_{m}$ are also singular (180). Substituting (180) into (201) we find

$$
\|H\|^{2}=(2 \pi)^{2} \gamma^{2 \beta} R_{r w}^{2}\left[\left|f_{l}\right|^{2}\left(1+\gamma^{2(\beta-1)} R_{r w}^{2} \hat{\Gamma}_{1}\left(R_{r w}^{2}\right)\right)^{2}+\sum_{m=2}^{\infty}\left|f_{m l}\right|^{2} \gamma^{2(m-1)(\beta-1)} R_{r w}^{2 m-2} \hat{\Gamma}_{m}^{2}\right]
$$

in this case we have determined that $\beta=1$ which yields

$$
\|H\|=2 \pi \gamma R_{r w}\left[\left|f_{l}\right|^{2}\left(1+R_{r w}^{2} \hat{\Gamma}_{1}\right)^{2}+\sum_{m=2}^{\infty}\left|f_{m l}\right|^{2} R_{r w}^{2 m-2} \hat{\Gamma}_{m}^{2}\right]^{1 / 2} \sim\left(K-K_{c}\right)^{1} .
$$


This is the scaling for $\|H\|$ Daido found for transitions at $l=1$ when there was no noise and the coupling had components at $l=2$. We obtain this result for a transition at arbitrary $l$ if the coupling satisfies $f_{2 l} \neq 0$ and $D=0$.

3. Suppose that $D=0$ and we have the special case with $f_{l} \neq 0, f_{2 l}=0$, and $f_{3 l} \neq 0$. In this case, our analysis of the recursion relations leaves considerable uncertainty in the value of $\beta$ as indicated by the range (190).

4. Suppose that $D=0$ and we have the special case with $\mathrm{O}(2)$ symmetry and also a coupling that satisfies $f(\phi)=-f(\phi+\pi / l)$. This requires $f_{n}=0$ unless $n$ is an odd multiple of $l$ and the non-zero components must be imaginary. In this case again one has typically $f_{l} \neq 0$ and $f_{3 l} \neq 0$, and our analysis of the recursion relations specifies $\beta$ only within the range (190). However this range nevertheless excludes the value $\beta=1 / 2$ Daido obtains for such symmetric systems. 13, 34, 35] The reason for this apparent discrepancy has not been found.

\section{Discussion}

Our analysis shares a key conclusion with Daido's study of scaling based on the order function. In the absence of noise, the presence of a coupling component $f_{2 l}$ at the first harmonic of the critical mode number $l$ will slow the onset of synchronized behavior so that the phaselocked component of the population scales like $\left(K-K_{c}\right)$ rather than $\left(K-K_{c}\right)^{1 / 2}$ as in the single-component coupling models. However, both approaches suffer a common deficiency; neither provides a simple explanation of why the onset of synchronization is slower if the coupling has a second harmonic component $\left(f_{2 l} \neq 0\right)$. This shift seems to be a true manybody effect requiring large $N$, but a dynamical mechanism has not been identified. Perhaps further numerical studies will shed some light on this issue. 
For a general coupling with $f_{2 l} \neq 0$, our calculations show two effects of adding noise to the phase dynamics. The diffusion term tends to suppress the linear instability so that $K_{c}$ increases, but in addition the noise prevents the poles from actually reaching the real axis and therefore removes the coefficient singularities; this modifies the scaling $\left(K-K_{c}\right)^{\beta}$ of the synchronized state changing $\beta$ from 1 to $1 / 2$. The interaction of these two effects raises the intriguing possiblity that under some circumstances, adding noise could actually yield an enhancement in the level of synchronization. In some recent studies of mean field oscillator models it has been noted that noise can enchance a collective oscillation. 36]

The linear stability analysis of section 2 clearly shows that for multi-component couplings one can expect multiple linear instabilities and this will allow for studies of the interaction between two different synchronizing transitions. In other settings the study of simultaneous linear instabilities, or codimension-two mode interactions, has been one of the keys to obtaining an analytical theory capable of describing the system beyond the threshold of the first instability. Such a theory can identify possibilities of secondary bifurcation and predict the appearance of states with nontrivial time-dependence. Despite the widespread interest in synchronization, and the large literature on the Kuramoto model, there has been no analysis of the codimension-two bifurcations that arise when more than one mode of synchronization is possible.

Although the justification for a phase model varies from one context to another, in the setting of coupled oscillators it requires two essential assumptions: weak coupling and a frequency spread $g(\omega)$ that is "sufficiently" small. [1, 2, 3n Moving beyond these assumptions necessitates restoring the dynamics of the oscillator amplitudes $r_{i}(t)$, and this adds an additional term to the kinetic equation of the schematic form

$$
\frac{\partial}{\partial r} \cdot(\dot{r} \rho)
$$


where $r=\left(r_{1}, \ldots, r_{N}\right)$ are the oscillator amplitudes and $\dot{r}$ is the non-stochastic component of the amplitude dynamics. In this situation, $\rho$ describes the joint distribution of oscillator phases and amplitudes. The effect of such a term on the synchronizing transition can be studied by analyzing the effect on the singularities of the nonlinear coefficients.

\section{Acknowledgements}

This work supported by NSF grant PHY-9423583.

\section{A Proof of Proposition 6.1}

Let $z_{0}=(\Omega+i \gamma) / l$ denote the root in $(107)$ - (108). If $m n=0$ then there is no pinching singularity and the integral in (111) has a finite limit; hence the limit is zero if $J>0$.

Assume $m n>0$, then for integrals with $m+n=2$ the limit can be evaluated from the Plemej formula [37] after a partial fraction expansion. The expansion isolates the singularity at $\gamma=0$,

$$
\int_{-\infty}^{\infty} \frac{d \omega \phi(\omega)}{\left(\omega-\alpha_{1}\right)\left(\omega-\beta_{1}^{*}\right)}=\frac{-i / \gamma}{\delta_{1}+\zeta_{1}+2 / l}\left[\int_{-\infty}^{\infty} \frac{d \omega \phi(\omega)}{\left(\omega-\alpha_{1}\right)}-\int_{-\infty}^{\infty} \frac{d \omega \phi(\omega)}{\left(\omega-\beta_{1}^{*}\right)}\right]
$$

and the limit (111) yields

$$
\begin{aligned}
\lim _{\gamma \rightarrow 0^{+}} \gamma\left|\int_{-\infty}^{\infty} \frac{d \omega \phi(\omega)}{\left(\omega-\alpha_{1}\right)\left(\omega-\beta_{1}^{*}\right)}\right| & =\frac{1}{\left(\delta_{1}+\zeta_{1}+2 / l\right)} \lim _{\gamma \rightarrow 0^{+}}\left|\int_{-\infty}^{\infty} \frac{d \omega \phi(\omega)}{\left(\omega-\alpha_{1}\right)}-\int_{-\infty}^{\infty} \frac{d \omega \phi(\omega)}{\left(\omega-\beta_{1}^{*}\right)}\right| \\
& =\frac{2 \pi\left|\phi\left(\Omega / l-K f_{0}\right)\right|}{\left(\delta_{1}+\zeta_{1}+2 / l\right)}
\end{aligned}
$$

The partial fraction expansion in (206) expresses an integrand of index 1 in terms of integrands of index 0. More generally, for integrands

$$
\int_{-\infty}^{\infty} d \omega \phi(\omega) \mathcal{G}(\omega)
$$


with index $m+n-1>1$, by expanding the integrand in partial fractions, they can be re-expressed as a sum of two integrands with index $m+n-2$ that are multiplied by an overall factor of $\gamma^{-1}$. Thus, if the partial fraction integrands of index $m+n-2$ satisfy (111) with $J=m+n-2$, then the original integrand will satisfy (111) with $J=m+n-1$; the value of $J$ must be incremented by one to allow for the factor of $\gamma^{-1}$.

A simple induction argument along these lines establishes (111) for all $m+n \geq 1$. In the limit in (111) is found to be non-zero in general so, barring an accidental cancellation, if $J$ is replaced by $J-1$ then the modified limit will diverge as $\gamma^{-1}$.

\section{B Derivation of the model}

Basically, the kinetic equation arises as the first member of a coupled hierarchy of equations quite similar to the BBGKY hierarchy well known in the kinetic theory of gases and this is probably the most elementary systematic approach. There are alternative derivations using path integral methods. 38

The solution of the system of $N$ stochastic differential equations in (1) is a Markov process with transition probability $\mathcal{P}\left(\theta, \omega, t \mid \theta^{\prime}, \omega^{\prime}, t^{\prime}\right)$ where $\theta=\left(\theta_{1}, \ldots, \theta_{N}\right)$ and $\omega=\left(\omega_{1}, \ldots, \omega_{N}\right)$. 39. This transition probability satisfies a linear Fokker-Planck equation

$$
\frac{\partial \mathcal{P}}{\partial t}+\frac{\partial}{\partial \theta} \cdot(\dot{\theta} \mathcal{P})=D \frac{\partial}{\partial \theta} \cdot \frac{\partial \mathcal{P}}{\partial \theta}
$$

subject to the initial condition

$$
\mathcal{P}\left(\theta, \omega, t^{\prime} \mid \theta^{\prime}, \omega^{\prime}, t^{\prime}\right)=\delta^{N}\left(\theta-\theta^{\prime}\right) \delta^{N}\left(\omega-\omega^{\prime}\right)
$$

The notation $\dot{\theta}=\left(\dot{\theta}_{1}, \ldots, \dot{\theta}_{N}\right)$ in (209) refers only to the non-stochastic part of the dynamics

$$
\dot{\theta}_{i}=\omega_{i}+\frac{K}{N} \sum_{j=1}^{N} f\left(\theta_{j}-\theta_{i}\right) .
$$


Given an initial normalized ensemble of populations $\mathcal{E}_{0}(\theta, \omega)$, the ensemble for $t>t_{0}$ is determined by

$$
\mathcal{E}(\theta, \omega, t)=\int d \omega^{\prime} \int d \theta^{\prime} \mathcal{P}\left(\theta, \omega, t \mid \theta^{\prime}, \omega^{\prime}, t_{0}\right) \mathcal{E}_{0}\left(\theta^{\prime}, \omega^{\prime}\right)
$$

equivalently, this is can be written as an evolution equation for $\mathcal{E}(\theta, \omega, t)$

$$
\frac{\partial \mathcal{E}}{\partial t}+\frac{\partial}{\partial \theta} \cdot(\dot{\theta} \mathcal{E})=D \frac{\partial}{\partial \theta} \cdot \frac{\partial \mathcal{E}}{\partial \theta}
$$

with initial condition $\mathcal{E}_{0}$. The original phase dynamics (11) is unchanged if we exchange a pair of oscillators $\left(\theta_{i}, \omega_{i}\right) \leftrightarrow\left(\theta_{j}, \omega_{j}\right)$ and it is natural to select initial ensembles $\mathcal{E}_{0}$ with this same invariance under pairwise exchange. In addition, for our problem, the values of the frequencies $\omega_{i}$ are assumed to obey a fixed distribution $g(\omega)$. This requirement necessitates a suitable choice of initial ensemble:

$$
\mathcal{E}_{0}(\theta, \omega)=G_{0}(\theta, \omega) \prod_{j=1}^{N} g\left(\omega_{j}\right)
$$

where the normalization of $\mathcal{E}_{0}(\theta, \omega)$ implies

$$
1=\int d \theta G_{0}(\theta, \omega)
$$

A reduced distribution $\rho_{s}$ for the ensemble is defined in the usual way

$$
\rho_{s}\left(\theta_{1}, \ldots, \theta_{s}, \omega_{1}, \ldots, \omega_{s}, t\right) \equiv \int d \theta_{s+1} \cdots d \theta_{N} \int d \omega_{s+1} \cdots d \omega_{N} \mathcal{E}(\theta, \omega, t)
$$

and one can derive a hierarchy of coupled equations expressing the evolution of $\rho_{s}$ in terms of $\rho_{s}$ and $\rho_{s+1}$ by performing the integration in (216) on the evolution equation (213). For our purposes it is sufficient to find the equation for $\rho_{1}$; integrating (213) over $\left(\theta_{2}, \ldots, \theta_{N}\right)$ and $\left(\omega_{2}, \ldots, \omega_{N}\right)$ yields

$$
\begin{array}{r}
\frac{\partial \rho_{1}}{\partial t}+\frac{\partial}{\partial \theta_{1}}\left[\left(\omega_{1}+K f(0) / N\right) \rho_{1}+\frac{K(N-1)}{N} \int d \theta_{2} \int d \omega_{2} f\left(\theta_{2}-\theta_{1}\right) \rho_{2}\left(\theta_{1}, \theta_{2}, \omega_{1}, \omega_{2}, t\right)\right] \\
=D \frac{\partial^{2} \rho_{1}}{\partial \theta_{1}^{2}} .(217)
\end{array}
$$


In terms of the two-oscillator correlation function,

$$
C\left(\theta_{1}, \theta_{2}, \omega_{1}, \omega_{2}, t\right) \equiv \rho_{1}\left(\theta_{1}, \omega_{1}, t\right) \rho_{1}\left(\theta_{2}, \omega_{2}, t\right)-\rho_{2}\left(\theta_{1}, \theta_{2}, \omega_{1}, \omega_{2}, t\right)
$$

this becomes

$$
\begin{aligned}
& \frac{\partial \rho_{1}}{\partial t}+\frac{\partial}{\partial \theta_{1}} {\left[\left(\omega_{1}+K f(0) / N\right) \rho_{1}+\frac{K(N-1) \rho_{1}}{N} \int d \theta_{2} \int d \omega_{2} f\left(\theta_{2}-\theta_{1}\right) \rho_{1}\left(\theta_{2}, \omega_{2}, t\right)\right]=} \\
& D \frac{\partial^{2} \rho_{1}}{\partial \theta_{1}^{2}}+\frac{\partial}{\partial \theta_{1}}\left[\frac{K(N-1)}{N} \int d \theta_{2} \int d \omega_{2} f\left(\theta_{2}-\theta_{1}\right) C\left(\theta_{1}, \theta_{2}, \omega_{1}, \omega_{2}, t\right)\right] \cdot
\end{aligned}
$$

We obtain an autonomous equation for $\rho_{1}$ by letting $N \rightarrow \infty$ and discarding the correlation term

$$
\frac{\partial \rho_{1}}{\partial t}+\frac{\partial}{\partial \theta_{1}}\left[\omega_{1} \rho_{1}+K \rho_{1} \int d \theta_{2} \int d \omega_{2} f\left(\theta_{2}-\theta_{1}\right) \rho_{1}\left(\theta_{2}, \omega_{2}, t\right)\right]=D \frac{\partial^{2} \rho_{1}}{\partial \theta_{1}^{2}}
$$

In some cases, one can rigorously prove that with a mean field interaction the effects of correlations are negligible in the limit $N \rightarrow \infty$. [40, 41, 38]) Whether this kind of result holds for the models considered here appears to be an unsettled issue. Finally, the ensemble choice in (214) implies that

$$
\int_{0}^{2 \pi} d \theta_{1} \rho_{1}\left(\theta_{1}, \omega_{1}, t_{0}\right)=g\left(\omega_{1}\right)
$$

so it is natural to define $\rho\left(\theta_{1}, \omega_{1}, t\right)$ by

$$
\rho_{1}\left(\theta_{1}, \omega_{1}, t\right) \equiv g\left(\omega_{1}\right) \rho\left(\theta_{1}, \omega_{1}, t\right)
$$

Substituting this expression for $\rho_{1}$ into (219) yields the kinetic equation (3) - (4).

It is interesting to note that the choice of initial ensemble (214) can be adapted to treat other forms of quenched randomness. [42] For example, we can allow for random variation in the coupling strength or even in the sign of the coupling between different pairs of phases. 


\section{References}

[1] G.B. Ermentrout and N. Kopell, Frequency plateaus in a chain of weakly coupled oscillators, I, SIAM J. Math Anal. 15 (1984) 215.

[2] Y. Kuramoto, Chemical Oscillations, Waves, and Turbulence, Springer-Verlag, New York (1984).

[3] P. Ashwin and J.W. Swift, The dynamics of $n$ weakly coupled identical oscillators, J. Nonlinear Sci. 269.

[4] A.T. Winfree, Biological rhythms and the behavior of populations of coupled oscillators, J. Theor. Biol. 16 (1967) 15-42.

[5] A.T. Winfree, Geometry of Biological Time, Springer-Verlag, New York (1990).

[6] S.H. Strogatz, Norbert Wiener's Brain Waves, in Lecture Notes in Biomathematics, Vol. 100, ed. S. Levin, (Springer Verlag, New York, 1994).

[7] C.M. Gray, P. Konig, A.K. Engel and W. Singer, Oscillatory responses in cat visual cortex exhibit inter-columnar synchronization which reflects global stimulus properties, Nature 338 (1989) 334-337.

[8] K. Wiesenfeld, P. Colet, S.H. Strogatz, Synchronization transitions in a disordered Josephson series array, Phys. Rev. Lett. 76 (1996) 404.

[9] J.W. Swift, S.H. Strogatz, and K. Wiesenfeld, Averaging of globally coupled oscillators, Phys. Rev. E 51 (1995) 1020.

[10] K. Wiesenfeld and J.W. Swift, Averaged equations for Josephson junction series arrays, Physica D 55 (1992) 239. 
[11] Y. Kuramoto and I. Nishikawa, Statistical macrodynamics of large dynamical systems. Case of a phase transition in oscillator communities, J. Stat. Phy. 49 (1987) 569-605.

[12] D Hansel, G. Mato, and C. Meunier, Phase dynamics for weakly coupled HodgkinHuxley neurons, Europhysics. Lett. 23 (1993) 367-372.

[13] H. Daido, Generic scaling at the onset of macroscopic mutual entrainment in limit-cycle oscillators with uniform all-to-all coupling, Phys. Rev. Lett. 73 (1994) 760-763.

[14] J.D. Crawford, Scaling and singularities in the entrainment of globally coupled oscillators, Phys. Rev. Lett. 74 (1995) 4341-4344.

[15] H. Daido, Onset of cooperative entrainment in limit-cycle oscillators with uniform allto-all interactions: bifurcation of the order function, Physica D 91 (1996) 24-66.

[16] H. Sakaguchi, Cooperative phenomena in coupled oscillator systems under external fields, Prog. Theor. Phys. 79 (1988) 39-46.

[17] S.H. Strogatz and R. Mirollo, Stability of incoherence in a population of coupled oscillators, J. Stat. Phys. 63 (1991) 613-635.

[18] S.H. Strogatz, R. Mirollo and P.C. Matthews, Coupled nonlinear oscillators below the synchronization threshold: relaxation by generalized Landau damping, Phys. Rev. Lett. 68 (1992) 2730-2733.

[19] J.D. Crawford, Amplitude expansions for instabilities in populations of globally-coupled oscillators, J. Stat. Phys 74 (1994) 1047-1084.

[20] J.D. Crawford, Introduction to bifurcation theory, Rev. Mod. Phys. 63 (1991) 991-1037. 
[21] A. Vanderbauwhede and G. Iooss, 1992, Centre manifold theory in infinite dimensions, Dynamics Reported, Vol. 1, Springer-Verlag, New York, 125-163.

[22] J.D. Crawford, Universal trapping scaling on the unstable manifold of a collisionless electrostatic mode, Phys. Rev. Lett. 73 (1994) 656-659.

[23] J.D. Crawford, Amplitude equations for electrostatic waves: universal singular behavior in the limit of weak instability, Phys. Plasmas 2 97-128 (1995).

[24] J.D. Crawford and A. Jayaraman, Nonlinear saturation of electrostatic waves: mobile ions modify trapping scaling, Phys. Rev. Lett. 773549 (1996).

[25] K. Case, Stability of inviscid plane Couette flow, Phys. Fl. 3143 (1960).

[26] R.J. Briggs, J.D. Daugherty, and R.H. Levy, Role of Landau damping in crossed-field electron beams and inviscid shear flow, Phys. Fl. 13 421-432 (1970).

[27] R. Pego and M.I. Weinstein, Phil. Trans. R. Soc. Lond. A 340 (1992) 47-94.

[28] R. Pego and M.I. Weinstein, in Differential Equations with Applications to Mathematical Physics, W.F. Ames, E.M. Harrell II, and J.V. Herod, eds., Academic Press, Orlando, 1993. pp. 273-286.

[29] R. Pego, P. Smereka, and M.I. Weinstein, Oscillatory instability of solitary waves in a continuum model of lattice vibrations, Nonlinearity, 8 921-941 (1995).

[30] G. Russo and P. Smereka, Kinetic theory for bubbly flow I: collisionless case, SIAM J. Appl. Math., 56 (1996).

[31] H. Daido, Critical conditions of macroscopic mutual entrainment in uniformly coupled limit-cycle oscillators, Prog. Theor. Phys. 89 (1993) 929-934. 
[32] J.D. Crawford and P.D. Hislop, Application of the method of spectral deformation to the Vlasov-Poisson system, Ann. Phys. 189 (1989) 265-317.

[33] M. Golubitsky, I. Stewart, and D.G. Schaeffer, Singularities and Groups in Bifurcation Theory: Vol. II, Appl. Math. Sci. 69, Springer-Verlag, New York (1988).

[34] H. Daido, Order function and macroscopic mutual entrainment in uniformly coupled limit-cycle oscillators, Prog. Theor. Phys. 88 (1992) 1213-1218.

[35] H. Daido, A solvable model of coupled limit-cycle oscillators exhibiting partial perfect synchrony and novel frequency spectra,, Physica D 69 (1993) 394-403.

[36] V. Hakim and W.-J. Rappel, Noise-induced periodic behavior in the globally coupled complex Ginzburg-Landau equation, Europhys. Lett. 27 (1994) 637.

[37] N. I. Muskhelishvili, Singular Integral Equations (Noordhoff, Groningen, The Netherlands, 1953), pp. 56-61.

[38] L.L. Bonilla, Stable nonequilibrium probability densities and phase transitions for meanfield models in the thermodynamic limit, J. Stat. Phys. 46 (1987) 659.

[39] C.R. Doering, Modelling complex systems: stochastic processes, stochastic differential equations, and Fokker-Planck equations, in Lectures in Complex Systems, Eds. L. Nadel and D. Stein, (Addison Wesley, New York, 1991), pp. 3-51.

[40] Rashmi C. Desai and Robert Zwanzig, Statistical mechanics of a nonlinear stochastic model, J. Stat. Phy. 19 (1978) 1-24.

[41] D.A. Dawson, Critical dynamics and fluctuations for a mean-field model of cooperative behavior, J. Stat. Phy. 31 (1983) 29. 
[42] L.L. Bonilla, Glassy synchronization in a population of coupled oscillators, J. Stat. Phys. 70 (1993) 921. 
Table 1: Order of calculation of $h_{m, j}(\omega)$ and $p_{j}$ from $\psi(\omega)$. The flow of calculation of the $h_{m, j}(\omega)$ is indicated by moving downward; the calculation of the $p_{j}$ proceeds by moving downward and to the left. For example, from $\psi(\omega), h_{2,0}$ can be calculated and then $p_{1}$ determined; $h_{1,0}$ and $h_{3,0}$ are calculated next from $h_{2,0}$ and then $h_{2,1}$ can be evaluated from $\left\{h_{1,0}, h_{3,0}, p_{1}\right\}$. This then determines $p_{2}$, and so forth. For $N \geq 2, p_{N}$ requires prior calculation of $h_{m, j}$ for $1 \leq m \leq N+1$ and $0 \leq j \leq N-m+1-2 \delta_{m, 1}$.

\begin{tabular}{l|ccccccc} 
& $m=1$ & $m=2$ & $m=3$ & $m=4$ & $m=5$ & $m=6$ & $\cdots$ \\
\hline$p_{0}$ & $\psi(\omega)$ & & & & & & \\
\hline$p_{1}$ & - & $h_{2,0}$ & & & & & \\
\hline$p_{2}$ & $h_{1,0}$ & & $h_{3,0}$ & & & & \\
\hline$p_{3}$ & $h_{1,1}$ & & & $h_{2,1}$ & & & \\
\hline$p_{4}$ & $h_{1,2}$ & $h_{2,2}$ & $h_{3,1}$ & & & & \\
\hline$p_{5}$ & $h_{1,3}$ & $h_{2,3}$ & $h_{3,2}$ & $h_{4,1}$ & & & \\
\hline & & $h_{2,4}$ & $h_{3,3}$ & $h_{4,2}$ & $h_{5,1}$ & & \\
$\vdots$ & & & & & & &
\end{tabular}

Table 2: Comparison of the exponent $\beta$ obtain from the amplitude equation with the exponent $\beta_{D}$ found from Daido's order function. We assume the synchronizing transition occurs at mode number $l$ for a coupling with $f_{l} \neq 0$. The bifurcation has $\mathrm{O}(2)$ symmetry when the coupling and frequency distribution satisfy $f(\phi)=-f(-\phi)$ and $g(\omega)=g(-\omega)$, respectively.

\begin{tabular}{cccccc} 
Noise & Symmetry & Coupling & $\beta$ & $\beta_{D}($ for $l=1)$ & Comment \\
\hline$D>0$ & $\mathrm{SO}(2)$ or $\mathrm{O}(2)$ & arbitrary & $1 / 2$ & - & generic case with noise \\
\hline$D=0$ & $\mathrm{SO}(2)$ or $\mathrm{O}(2)$ & $f_{2 l} \neq 0$ & 1 & 1 & generic case without noise \\
\hline$D=0$ & $\mathrm{SO}(2)$ or $\mathrm{O}(2)$ & $f_{2 l}=0, f_{3 l} \neq 0$ & {$[3 / 4,1]$} & - & \\
\hline$D=0$ & $\mathrm{O}(2)$ & $f(\phi)=-f(\phi+\pi / l)$ & {$[3 / 4,1]$} & $1 / 2$ & $\beta$ and $\beta_{D}$ differ \\
\hline$D=0$ & $\mathrm{SO}(2)$ or $\mathrm{O}(2)$ & $f_{m l}=0, m>1$ & $1 / 2$ & $1 / 2$ & single component case \\
\hline
\end{tabular}

